\title{
Active rheology and anticommensuration effects for driven probe particles on two-dimensional periodic pinning substrates
}

\author{
C. J. O. Reichhardt $\odot$ and C. Reichhardt \\ Theoretical Division and Center for Nonlinear Studies, Los Alamos National Laboratory, Los Alamos, New Mexico 87545, USA
}

(Received 10 December 2021; accepted 24 February 2022; published 8 March 2022)

\begin{abstract}
For an assembly of particles interacting with a two-dimensional periodic substrate, a series of commensuration effects can arise when the number of particles is an integer multiple of the number of substrate minima. Such commensuration effects can appear for vortices in type-II superconductors with periodic pinning or for colloidal particles on optical landscapes. Under bulk external driving, the pinning or drag on the particles is strongly enhanced at commensuration. Here we consider the active rheology of a single particle driven through an assembly of particles coupled to a periodic substrate at different commensurate conditions. For increasing density at fixed driving force, we observe nonmonotonic drag along with what we call an anticommensuration effect where the drag or pinning effectiveness is reduced in commensurate states, opposite from the behavior typically observed under bulk driving. The velocity enhancement or drag reduction appears when the background particles form a crystalline state that is coupled more strongly to the substrate than to the driven particle, while under incommensurate conditions, the background particles are disordered and produce enhanced drag on the probe particle. The velocity noise of the driven particle has a narrow band signature at commensuration and a broad band signature away from commensuration. We map out the regions in which viscous flow, periodic flow, and a pinned phase appear. We show that the effects we observe are robust on both square and triangular substrate arrays and for both vortices in type-II superconductors and colloidal particles on optical landscapes.
\end{abstract}

DOI: 10.1103/PhysRevResearch.4.013190

\section{INTRODUCTION}

A wide variety of systems can be described in terms of a collection of interacting particles coupled to a periodic substrate. Commensuration effects arise when the number of particles is an integer or rational fractional multiple of the number of substrate minima and the system forms a highly ordered crystalline state [1-9]. At incommensurate fillings, the particles can remain in a lattice that floats above a weak substrate, or the particle positions can be disordered by a stronger substrate; in each case, the effectiveness of the pinning is reduced [1,6,7,9]. For fillings just outside of commensuration, the system can be mostly ordered and contain a small number of localized excitations or solitons [8,9]. Under an applied drive, at commensurate conditions the depinning threshold above which motion occurs shifts to higher drives, while for incommensurate conditions, the depinning threshold is depressed or can show distinct steps due to the separate depinning of the solitons and the bulk particles; additionally, if the particles are already moving, the velocity is strongly suppressed at commensuration and is largest for incommensurate conditions [8-12]. The ordering of the particles at commensuration depends on the dimensionality and

Published by the American Physical Society under the terms of the Creative Commons Attribution 4.0 International license. Further distribution of this work must maintain attribution to the author $(s)$ and the published article's title, journal citation, and DOI. geometry of the periodic substrate. For a two-dimensional (2D) particle assembly on a quasi-one-dimensional (q1D) substrate, smooth transitions occur between ordered commensurate and disordered incommensurate states, such as crystal and smectic states [13-15], while for a 2D system with a 2D periodic substrate, the commensurate effects can be sharper and consist of transitions between various types of 2D crystals [2,4-6,8,9,16-19]. Examples of 2D particle systems coupled to a $2 \mathrm{D}$ periodic substrate include vortices in type-II superconductors with periodic pinning, [2,9-11,16,17,20-27], vortices in Bose-Einstein condensates with optical traps [5], colloidal particles on optical traps $[8,18,19,19,28,29]$ or etched surfaces $[6,12,30,31]$, active matter on patterned substrates [32], cold atom systems [33], dusty plasmas on 2D arrays [34], skyrmions in nanostructured samples [35,36], coupled nanomagnetic islands [37], and numerous types of atomic or frictional systems [7,38-40]. In many of these systems, when driving is applied, dynamic transitions can produce signatures in the velocity-force curves [7-12,24,38,40,41] and structural changes can occur in the particle positions at the depinning threshold or for higher drives [9,12,24,31,41,42]. The effect of varied commensuration conditions on pinning has been heavily studied for vortices in type-II superconductors, where the ratio of the number of particles to the number of pinning sites can be varied easily by changing the magnetic field, and where peaks in the critical currents or reduced vortex velocities appear at the matching fields [9-11,16,17,20,22,2527,43] and at fractional matching fillings [21-24]. Peaks in the depinning force at commensurate fillings have also been studied for colloidal systems $[6,8,12]$. 
In studies of depinning effects at commensurate and incommensurate fields in the systems above, the driving was applied to all of the particles at the same time; however, in many systems it is possible to drive only a single individual particle through the sample. Measurement of the dynamics of a single driven probe particle though a medium of other background particles is known as active rheology [44-50]. Here, an individual particle is dragged under different conditions such as constant force, constant velocity, or increasing force, and the resulting pinning threshold or viscous drag can be measured as the system parameters are varied. In colloidal assemblies, active rheology has been applied to glassy systems $[45,49,51-53]$, where there can be an onset of a finite depinning threshold or a rapid increase in the drag as the glass transition is approached. For granular systems, investigations have revealed how the drag, pinning, and fluctuations on an individual driven grain change as the jamming transition is approached, along with the range out to which the surrounding particles become disordered as the driven particle moves through the sample [54-56]. Other studies have revealed the changes in the motion [57] or the viscoelastic response [58] of the driven particle as a transition occurs from elastic to plastic distortions in the surrounding medium. For systems such as superconducting vortices or magnetic skyrmions, individual driven particles can interact both with the other particles and also with a substrate in the form of a pinning landscape [59-63]. The most studied example of this involves the dragging of individual vortices in order to examine vortex-pinning and vortex-vortex interactions [59-61,64-67]. The depinning threshold for the individually driven vortex can be nonzero even in the absence of random pinning due to the elasticity of the surrounding vortex lattice, while when random pinning is added to the system, the depinning threshold is enhanced [68].

For individually dragged vortices moving over periodic pinning arrays containing pinned vortices, stick-slip motion can appear, and the dynamics of the driven vortex can vary depending on the orientation of the driving direction with respect to the symmetry of the underlying pinning array [69]. It has also been proposed that for individually driven vortices on a periodic pinning array in a system which hosts Majorana fermions, it is possible to take advantage of the different dynamics to create various types of quantum logic gates [70]. An open question is how the dynamics or drag on a driven particle such as a superconducting vortex changes as the system is tuned from an incommensurate to a commensurate state. One possibility is that the drag will be enhanced at matching, as found in bulk driven systems.

Although active rheology has been studied extensively for systems in the absence of a substrate, far less is known about when happens when a periodic substrate is present. Here we examine superconducting vortices and colloidal particles interacting with $2 \mathrm{D}$ square and triangular pinning arrays, where we drive a single particle through the medium for varied filling fractions and driving forces. At a constant driving force and in the absence of pinning, the mobility of the probe particle decreases monotonically with increasing system density due to increased collisions with the background particles; however, when the periodic pinning array is present, a series of velocity peaks appear at commensurate fillings when the system forms an ordered lattice. We call these peaks anticommenensuration effects since for bulk driving in most systems with pinning, the velocity of the particles is reduced at the matching conditions. For our single driven particle, the velocity enhancement arises when the surrounding particles become more strongly coupled to the substrate than to the probe particle under a matching condition. At incommensurate fields where the system is disordered, the probe particle interacts more strongly with the background particles and experiences a higher effective drag force. At a matching condition, the velocity of the probe particle exhibits a periodic or narrow band noise signature, while at incommensurate fillings, the probe particle motion is disordered or chaotic, giving rise to a broad band noise signal. We also find that the pinning effect on the probe particle is reduced at the matching conditions. As function of pinning force and filling fraction, we map out the pinned, viscous flow, and ordered flow phases. In general, under a constant drive the probe particle moves at a higher velocity in the presence of pinning than in the absence of pinning since the background particles cannot be entrained by the probe particle when they are trapped by pinning sites. In the case of colloidal particles, as the effective charge of the particles is reduced, we observe a transition from a pinned state to an unpinned state. This is the opposite of the behavior found in bulk driven systems, where a weakening of the colloid-colloid interactions causes the colloids to become more strongly coupled to the substrate.

\section{SIMULATION AND SYSTEM}

We consider a two-dimensional system containing $N$ particles along with a periodic array of $N_{p}$ pinning sites in the form of potential energy minima that can each capture a single particle. A matching condition occurs when the ratio $f=N / N_{p}$ is an integer, and it is known from previous studies of superconducting vortices and colloidal particles that at such fillings, the particles form an ordered crystal state $[2,5,6,8,9,12,16$ 18]. At incommensurate fillings, the system is disordered or contains localized regions of excitations. It is also possible for ordered crystalline states to appear at certain fractional matching conditions such as $f=1 / 2$ on a square pinning array or $f=1 / 3$ on a triangular pinning array $[21,22,24]$. In the first part of the work, we focus on vortices in type-II superconductors with periodic pinning, where there has been extensive numerical modeling $[9,17,22,24,42,69,70]$ and experimental studies $[2,9,16,20,21]$ examining the commensuration effects under bulk driving. Here we instead drive only a single vortex. The equation of motion for vortex $i$ is given by

$$
\alpha_{d} \mathbf{v}_{i}=\mathbf{F}_{i}^{v v}+\mathbf{F}_{i}^{p}+\mathbf{F}_{i}^{D},
$$

where $\mathbf{v}_{i}=d \mathbf{r}_{i} / d t$ is the velocity and $\mathbf{r}_{i}$ is the position of vortex $i$. The dynamics is overdamped and we set the damping constant $\alpha_{d}$ equal to unity. Vortex-vortex interactions are described by the term $\mathbf{F}_{i}^{v v}=\sum_{j=1}^{N} K_{1}\left(r_{i j}\right) \hat{\mathbf{r}}_{i j}$, where $r_{i j}=\mid \mathbf{r}_{i}$ $\mathbf{r}_{j} \mid, \hat{\mathbf{r}}_{i j}=\left(\mathbf{r}_{i}-\mathbf{r}_{j}\right) / r_{i j}$, and $K_{1}$ is the modified Bessel function which decays exponentially for large $r$. Pinning sites are modeled as parabolic traps with a maximum range of $r_{p}$ that produce a pinning force described by $\mathbf{F}_{i}^{p}=\sum_{k=1}^{N_{p}}\left(F_{p} / r_{p}\right)\left(\mathbf{r}_{i}-\right.$ $\left.\mathbf{r}_{k}^{(p)}\right) \Theta\left(r_{p}-\left|\mathbf{r}_{i}-\mathbf{r}_{k}^{(p)}\right|\right)$. Here $F_{p}$ is the maximum pinning force and $\Theta$ is the Heaviside step function. A driving force $\mathbf{F}^{D}=F_{D} \hat{\mathbf{x}}$ is applied to only one vortex with $i=D$ and is 
always aligned with the $x$ direction; the driving force on all of the other vortices with $i \neq D$ is set to zero.

For the driven or probe vortex, we measure the net velocity $V=\mathbf{v}_{i=D} \cdot \hat{\mathbf{x}}$. At a given driving force, we consider both time series data and the time averaged velocity $\langle V\rangle=$ $\tau^{-1} \sum_{t_{i}=1}^{\tau} V\left(t_{i}\right)$, where the averaging time $\tau=1 \times 10^{5}$ simulation time steps. We consider only a single realization for each parameter set and do not average over realizations. In some cases, we apply a constant drive, while in other cases we sweep the driving force from zero to a maximum value in order to measure the depinning force $F_{c}$ and the effective drag. The initial positions of the vortices are obtained using simulated annealing [17], where the sample is initialized at a high temperature using random Langevin kicks and gradually cooled to zero in order to obtain a low energy state. This process is repeated to obtain starting configurations for different filling factors $f$. In superconducting systems, the vortex density is proportional to the magnetic field $B$ and the filling factor is measured relative to the $1: 1$ vortex to pinning site matching condition known as the matching field $B_{\phi}$, such that $f=B / B_{\phi}$. The sample is of size $L \times L$ with $L=36 \lambda$, where $\lambda$ is the London penetration depth, and has periodic boundary conditions in the $x$ and $y$ directions. Note that according to standard simulation practice, for a square pinning lattice, the sample is of equal size in the $x$ and $y$ directions, but for a triangular pinning lattice, the length of the sample along the $y$ direction is reduced slightly in order to accommodate a triangular lattice without any defects. We fix the pinning density to $n_{p}=N_{p} / L^{2}=0.5$ and the pinning radius to $r_{p}=$ 0.35 . We have previously used these parameters to investigate commensuration effects for bulk driven vortices, where peaks in the critical depinning force appear at commensurate fields $[17,24,71,72]$.

For the colloidal system, we use similar overdamped dynamics but replace the Bessel function interaction by a screened Coulomb or Yukawa potential of the form $U\left(r_{i j}\right) \propto$ $Q^{2} \exp \left(-\kappa r_{i j}\right) / r_{i j}$, where $Q$ is the charge on an individual colloidal particle $[18,19]$. Here, the strength of the colloidcolloid interaction can be varied by changing the charge $Q$, such as by modifying the ion concentration of the solution.

Regarding our choice of notation for the filling fraction $f$, in most of this work we use the expression $f=B / B_{\phi}$ since this notation is commonly employed in the superconducting vortex literature. In the simulation we vary $B / B_{\phi}$ by changing the number of vortices $N$ in the sample. For the square pinning array, there are always $N_{p}=625$ pinning sites, and for the triangular pinning array there are always $N_{p}=648$ pinning sites, so the plots can also be read by substituting " $N / N_{p}$ " for " $B / B_{\phi}$ " since the tick marks will be unchanged.

\section{RESULTS}

We first consider a superconducting system with a single driven vortex. In Fig. 1, we plot a subsection of the sample showing the positions of the bulk vortices and pinning sites along with the trajectory of the driven vortex at $f=B / B_{\phi}=$ 1.0 and $F_{p}=0.25$. Here the bulk vortices form an ordered commensurate state in which each pinning site captures one vortex and the driven probe particle moves along a 1D channel in the interstitial region.

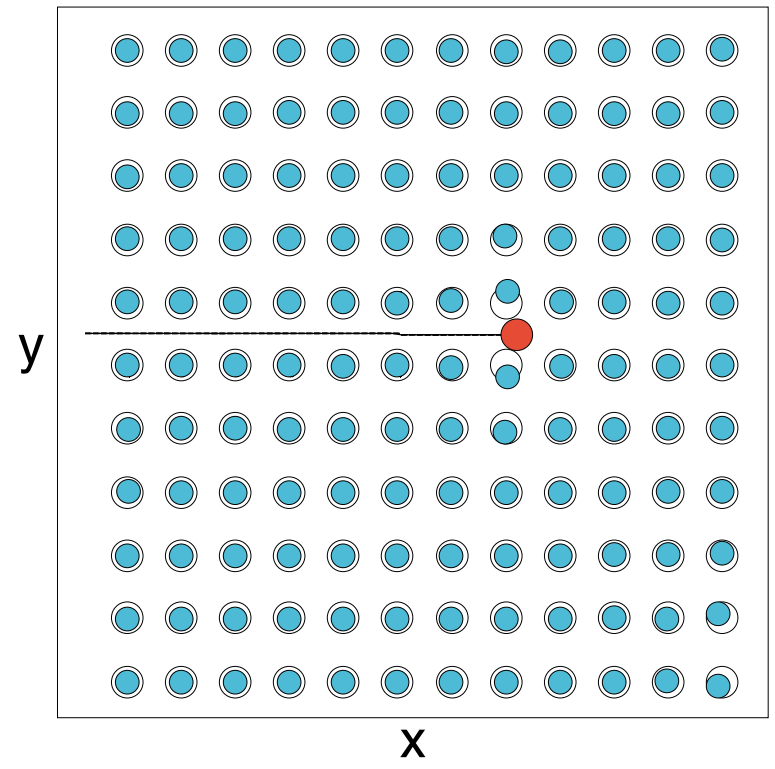

FIG. 1. Image of a subsection of the superconducting vortex system showing a square pinning array (open circles), the bulk vortices (blue circles), the driven vortex (red circle), and the vortex trajectories (lines) in a sample with $f=B / B_{\phi}=1.0$ or a $1: 1$ matching of bulk vortices to pinning sites. Here, $F_{D}=1.0$ and $F_{p}=0.25$. The size of the pinning sites has been adjusted for clarity.

In Fig. 2(a), we plot the average velocity of the driven particle $\langle V\rangle$ at an applied drive of $F_{D}=1.0$ as a function of $B / B_{\phi}$ for a square pinning array with $F_{p}=0.0$ (no pinning) and $F_{p}=0.03$. When there is no pinning, $\langle V\rangle$ decreases monotonically approximately as $\langle V\rangle \propto B^{1 / 2}$ from $\langle V\rangle=1.0$ at $B / B_{\phi}=0.0$ to $\langle V\rangle=0.3$ at $B / B_{\phi}=6.0$. This behavior arises due to the increased number and strength of interactions between the driven vortex and the background vortices as the magnetic field, and thus the vortex density, increases. In the presence of a square pinning array with $F_{p}=$ 0.03 , small peaks appear in $\langle V\rangle$ at $B / B_{\phi}=1.0,3.0$, and 6.0, along with a very weak peak at $B / B_{\phi}=4.0$. At these fillings, the system forms an ordered lattice. From previous studies of vortices in a square periodic pinning array, it is known that ordered lattices appear only for certain matching fields and not for all matching fields, while the particle ordering also depends on the pinning strength [17]. Specifically, for a square pinning array of sufficiently strong pinning sites, a square vortex lattice is stabilized at $B / B_{\phi}=1.0,2.0$, and 5.0, a triangular vortex lattice appears at $B / B_{\phi}=4.0$ and 6.0, and peaks in the depinning force occur at all of these fields under bulk driving [17]. For the square pinning lattice with $F_{p}=0.03$ in Fig. 2(a), the pinning is not strong enough to stabilize a square lattice at $B / B_{\phi}=2.0$ and a floating distorted triangular lattice appears instead. This lattice is weakly pinned so there is no peak in $\langle V\rangle$ at this filling. Triangular vortex orderings occur at $B / B_{\phi}=3.0$ and 6.0 , giving sharp peaks in $\langle V\rangle$, while there is only unstable triangular ordering at $B / B_{\phi}=4.0$, producing a weak peak. In Fig. 2(b), we plot $\langle V\rangle$ versus $B / B_{\phi}$ for a square pinning array with a stronger pinning force of $F_{p}=0.25$ where the orderings are more similar to those found in previous studies [17], with a vortex lattice that 


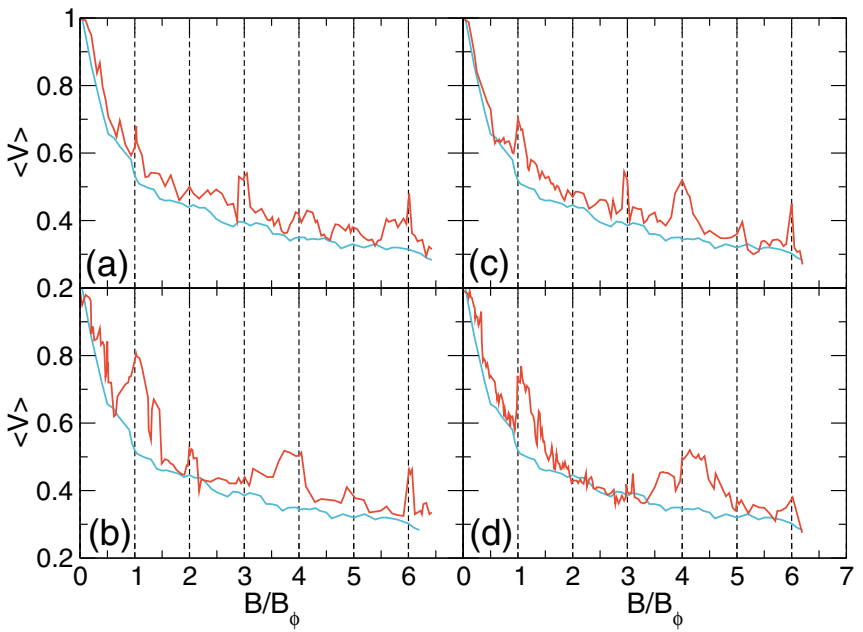

FIG. 2. (a) The average velocity $\langle V\rangle$ of the driven particle versus $B / B_{\phi}$ at $F_{D}=1.0$. The blue line is the same in all panels and is from a sample with $F_{p}=0.0$ or no pinning, which shows a monotonic decrease in $\langle V\rangle$ with increasing $B / B_{\phi}$. The red lines are for finite pinning. (a) A square pinning array with $F_{p}=0.03$. (b) A square pinning array with $F_{p}=0.25$, where there are velocity peaks at $B / B_{\phi}=1 / 2$, $1.0,2.0,4.0$, and 6.0, along with weaker peaks at $B / B_{\phi}=2.0$ and 5.0. (c) A triangular pinning array with $F_{p}=0.03$, where there are velocity peaks at $B / B_{\phi}=1.0,3.0,4.0$, and 6.0 , as well as a missing peak at $B / B_{\phi}=2.0$. (d) A triangular pinning array with $F_{p}=0.25$. In general, the samples containing finite pinning also have a larger average $\langle V\rangle$ compared to samples without pinning.

is square at $B / B_{\phi}=1.0$ and 2.0 and triangular at $B / B_{\phi}=4.0$ and 6.0. At $B / B_{\phi}=3.0$, the vortex lattice is neither square nor triangular, but has dimer ordering [17], while at $B / B_{\phi}=5.0$, the vortex lattice is mostly square but contains numerous grain boundaries. Fields with square or triangular vortex lattices are accompanied by a peak in the vortex velocity, and there is a missing velocity peak at $B / B_{\phi}=3.0$. In previous work with bulk driven systems, the formation of a square vortex lattice under the influence of strong pinning is correlated with the appearance of peaks in the critical depinning force and a reduction in the velocity of flowing vortices. There was also a missing peak in the depinning force at $B / B_{\phi}=3.0$. The previous work combined with our new results indicates that the formation of ordered vortex lattices at commensurate fields produces enhanced pinning (indicated by a peak in the critical depinning and a dip in the vortex velocity) for bulk driven systems but reduced pinning (indicated by a peak in the vortex velocity) for individually driven vortices. We note that there is also a submatching velocity peak at $B / B_{\phi}=1 / 2$ in Fig. 2(b).

In Fig. 2(c), we plot $\langle V\rangle$ versus $B / B_{\phi}$ for the pin-free sample and for a sample containing a weak triangular pinning lattice with $F_{p}=0.03$. In the presence of pinning, peaks in the velocity appear at $B / B_{\phi}=1.0,3.0,4.0$, and 6.0 that are more prominent than the peaks observed for a square pinning lattice. In previous work on bulk driven vortices, commensurate effects produce enhanced pinning (rather than enhanced velocity) at $B / B_{\phi}=1.0,3.0$, and 4.0 , but not at $B / B_{\phi}=2.0$, since for the latter filling the vortices form a partially ordered honeycomb lattice rather than a triangular lattice. For the
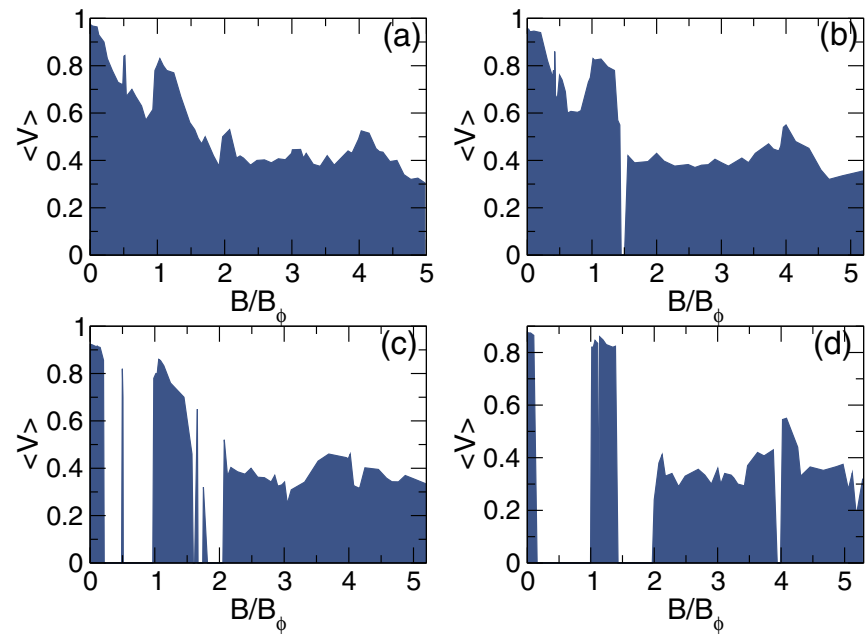

FIG. 3. $\langle V\rangle$ versus $B / B_{\phi}$ for the system in Fig. 2(a) with a square pinning array at $F_{D}=1.0$ and (a) $F_{p}=0.375$, (b) 0.5 , (c) 0.625 , and (d) 0.75 . Here the commensurate conditions correspond to fields at which there is a peak in the velocity. For larger $F_{p}$, there are windows of field over which the system becomes pinned with $\langle V\rangle=0$.

individually driven vortex, Fig. 2 indicates that the net velocity is generally lower in the absence of pinning and higher in the presence of pinning, which is the opposite of the behavior generally found for bulk driving. Additionally, the peak velocity is shifted slightly above the matching field rather than occurring exactly at the matching field. In Fig. 2(d), we plot $\langle V\rangle$ versus $B / B_{\phi}$ for a stronger triangular pinning array with $F_{p}=0.25$. Here there is a broad velocity enhancement peak over the range $3.0<B / B_{\phi}<4.5$, where the system forms a triangular lattice with varied orientations.

The overall behavior in Fig. 2 is the opposite of what is found for bulk driving, where at commensurate fillings the driven particles are less coupled to the substrate and move more rapidly. The drag on an individually driven particle arises due to interactions with the surrounding background particles, and for incommensurate fillings, these particles are more disordered and less coupled to the substrate, permitting them to couple more effectively to the driven particle and generate a larger drag effect. When pinning is present, the background particles couple to the substrate and cannot be entrained as easily by the driven particle, which reduces the drag. Under commensurate conditions, the surrounding particles are very strongly coupled to the substrate and poorly coupled to the driven particle. As a result, the driven particle is more mobile at the matching fields, which we call an anticommensuration effect. The peaks in the velocity fall at fields slightly above matching because just below matching, the background particles form a commensurate lattice with vacancies that act as effective trapping sites for the driven particle. In contrast, slightly above commensuration, there are some interstitials in the commensurate configuration which are unable to trap the driven particle.

In Fig. 3, we plot $\langle V\rangle$ versus $F_{D}$ for the system in Fig. 2(a,b) with a square pinning lattice at different pinning strengths. At $F_{p}=0.375$ in Fig. 3(a), strong velocity peaks appear at $B / B_{\phi}=1 / 2,1.0,2.0$, and 4.0, while for $F_{p}=0.5$ in Fig. 3(b), 

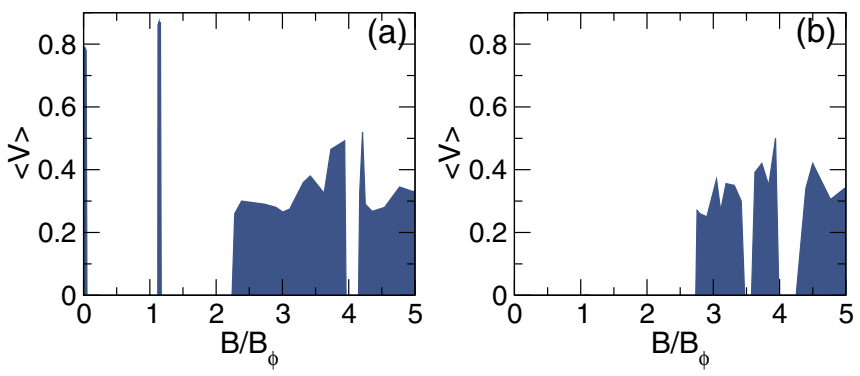

FIG. 4. $\langle V\rangle$ versus $B / B_{\phi}$ for the system in Fig. 2(a) with a square pinning array at $F_{D}=1.0$ at (a) $F_{p}=0.875$ and (b) $F_{p}=1.0$, where the widths of the pinned regions increase with increasing $F_{p}$.

the peak locations remain robust but several regions have opened in which the driven particle is pinned with a velocity of $\langle V\rangle=0$. One pinned region appears just above $B / B_{\phi}=1 / 2$, while another is found near $B / B_{\phi}=1.5$. For $F_{p}=0.625$ in Fig. 3(c), there are a growing number of pinned regions, while peaks in $\langle V\rangle$ appear at $B / B_{\phi}=1 / 2,1.0$, and just above 2.0. For $F_{p}=0.75$ in Fig. $3(\mathrm{~d})$, the pinned regions have expanded while velocity peaks persist at $B / B_{\phi}=1.2$ and just above $B / B_{\phi}=2.0$ and $B / B_{\phi}=4.0$. These results indicate that strong pinning effectiveness appears in the incommensurate phase, which is the opposite of the behavior observed in a bulk driven system. Since $F_{D}>F_{p}$ for all of the samples in Fig. 3, the effective drag or pinning of the driven vortex is produced by a combination of trapping directly at pinning sites and interactions with background pinned vortices.

In Figs. 4(a) and $4(\mathrm{~b})$, we show $\langle V\rangle$ versus $B / B_{\phi}$ for samples with a square pinning array at higher pinning strengths of $F_{p}=0.875$ and 1.0. At $F_{p}=0.875$ in Fig. $4(\mathrm{a})$, there is a small region close to $B / B_{\phi}=0.0$ where the driven vortex only interacts with the pinning sites and remains unpinned since $F_{D}>F_{p}$. In this regime, $\langle V\rangle=0.79<F_{D}$ since the driven particle is still slowed by the interactions with the pinning sites. Just above $B / B_{\phi}=1.0$, we find another pinned region which extends up to $B / B_{\phi}=2.5$. For a higher $F_{p}=1.0$ in Fig. 4(b), individual pinning sites can trap the driven vortex since we now have $F_{p}=F_{D}$, and a large pinned regime extends from $0 \leqslant B / B_{\phi}<2.75$.

To characterize the dynamics of the driven particle, we examine detailed velocity time series as well as images of the sample. In Fig. 5(a), we illustrate the system in Fig. 3(a) at $F_{p}=0.375$ for a low field of $B / B_{\phi}=0.05$, where the driven particle motion is localized along a single row of pinning, and there are only weak interactions with the background vortices. In Fig. 6(a), we plot the time series of the velocity $V(t)$ for the sample in Fig. 5(a). The signal is periodic since the driven vortex moves directly over the pinning sites in a single row, while there are modulations of the signal at longer time scales due to the occasional interactions with the background vortices. Figure 6(b) shows the Fourier transform $F(\omega)$ of the time series in Fig. 6(a),

$$
F(\omega)=\int V(t) e^{-i \omega t} d t .
$$

Here $F(\omega)$ exhibits a strong periodic or narrow band noise feature due to the periodic motion of the driven vortex.

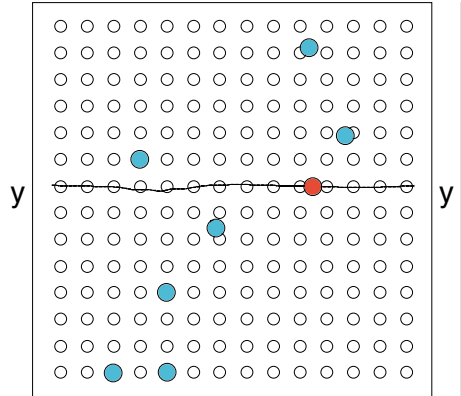

(a)

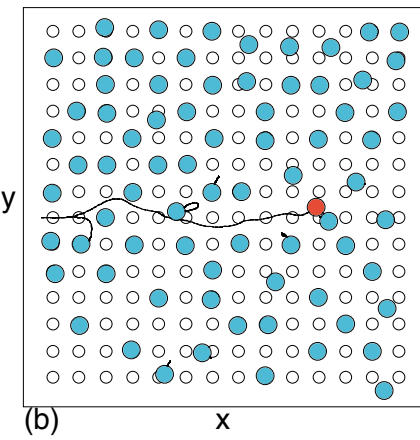

FIG. 5. Image of a subsection of the superconducting vortex system with a square pinning array (open circles), bulk vortices (blue circles), the driven vortex (red circle), and the vortex trajectories (lines) for the system in Fig. 3(a) with $F_{p}=0.375$ and $F_{D}=1.0$. The size of the pinning sites has been adjusted for clarity. (a) $B / B_{\phi}=0.05$ where the driven particle moves along a pinning row and has little interaction with the other vortices. (b) $B / B_{\phi}=0.414$, an incommensurate regime, where the flow is much more disordered with multiple collisions occurring between the driven vortex and the background vortices.

Figure 5(b) shows the vortex trajectories at $B / B_{\phi}=0.414$ in the incommensurate region, where the motion of the driven particle is much more random due to strong interactions with numerous background vortices, which produce considerable random winding of the trajectory in the direction transverse to the drive. The driven vortex follows a different trajectory each time it passes through the periodic boundary conditions, suggesting that the flow has a chaotic character. The corresponding velocity time series in Fig. 6(c) contains strong random fluctuations, while the Fourier transform $F(\omega)$ in Fig. 6(d) is broad and has lost the sharp peaks observed at $B / B_{\phi}=0.05$. When $B / B_{\phi}=0.5$, the background vortices adopt an ordered checkerboard ordering [21,24], and the
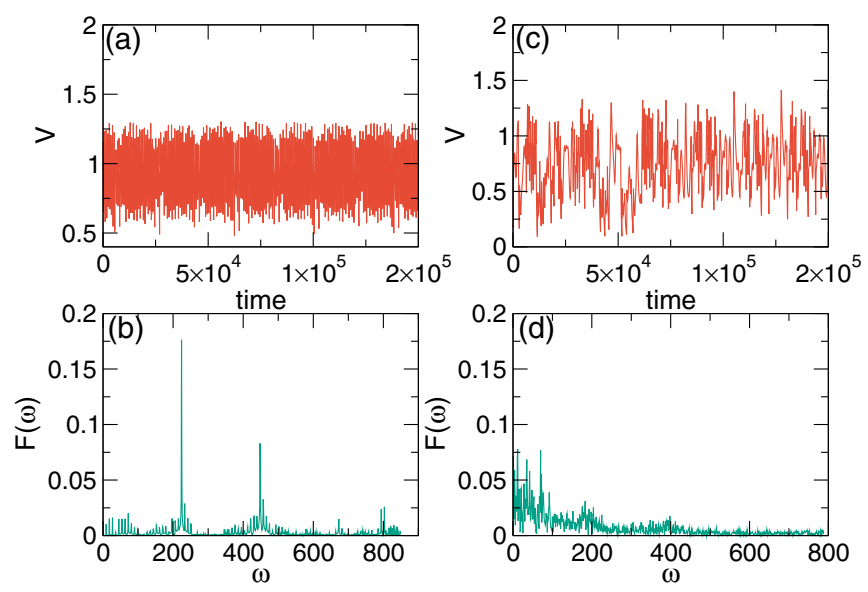

FIG. 6. [(a) and (c)] Time series of the driven particle velocity $V$ and [(b) and (d)] the corresponding Fourier transform $F(\omega)$ for the system in Fig. 5 with a square pinning array at $F_{p}=0.375$ and $F_{D}=1.0$. [(a) and (b)] At $B / B_{\phi}=0.05$, the motion is periodic, producing a series of peaks in $S(\omega)$. [(c) and (d)] At $B / B_{\phi}=0.414$, the velocity signal is more disordered and the corresponding $F(\omega)$ has much broader noise features. 


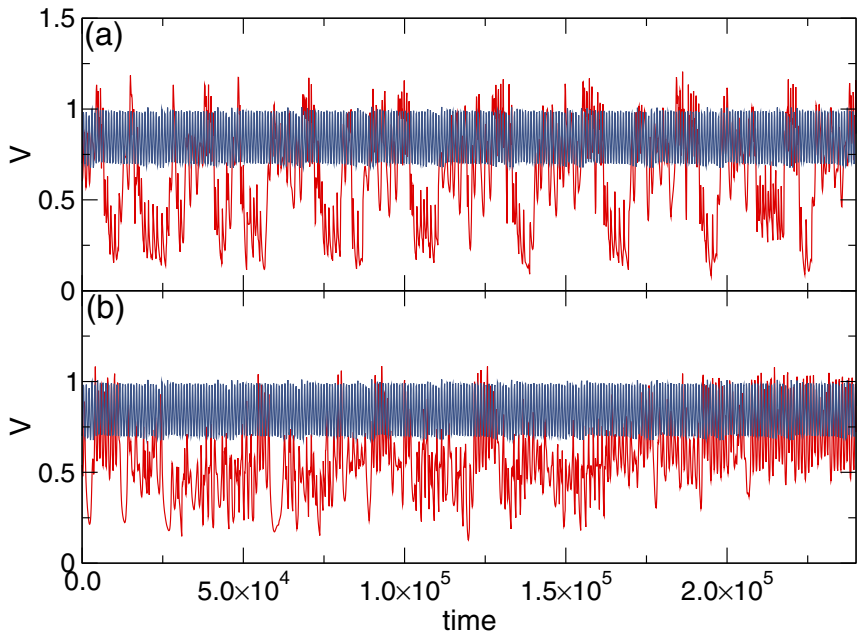

FIG. 7. (a) Time series of the velocity $V$ of the driven particle for the system from Fig. 1 with $F_{p}=0.375$ and $F_{D}=1.0$ at $B / B_{\phi}=1.0$ (blue) and $B / B_{\phi}=0.828$ (red). (b) The same for $B / B_{\phi}=1.0$ (blue) and $B / B_{\phi}=1.55$ (red). In each case, the velocity at the commensurate filling of $B / B_{\phi}=1$ is periodic and has a higher average value than the velocity at the incommensurate fillings.

driven particle moves along an ordered 1D path without collisions, similar to the trajectory shown in Fig. 1.

To illustrate more clearly the change in the velocity fluctuations of the driven particle depending upon whether the filling is commensurate or not, in Fig. 7(a), we plot a time series of the velocity $V$ of the driven particle for the system from Fig. 1 with $F_{p}=0.375$ and $F_{D}=1.0$ at a commensurate state of $B / B_{\phi}=1.0$ and an incommensurate state of $B / B_{\phi}=0.828$. For the incommensurate field, the average velocity is lower and the signal is more disordered, while for $B / B_{\phi}=1.0$, the signal is periodic and has a higher average value. At $B / B_{\phi}=1.0$, the driven particle moves in a $1 \mathrm{D}$ path as illustrated in Fig. 1, and the oscillatory part of the velocity signal arises from the periodic interactions of the driven particle with the vortices at the pinning sites. Even though there are more background vortices with which the driven particle could interact at $B / B_{\phi}=1.0$ than at $B / B_{\phi}=0.828$, the velocity is higher for $B / B_{\phi}=1.0$ since the ordered nature of the commensurate flow reduces the number of effective collisions between the driven particle and the background vortices. In Fig. 7(b), we compare the velocity time series in the same sample at $B / B_{\phi}=1.0$ with that of the higher incommensurate field $B / B_{\phi}=1.55$. Here, the velocity is again reduced in magnitude and more chaotic in nature at the incommensurate filling than at the $1: 1$ matching field.

In Fig. 8, we show the power spectrum $S(\omega)$, which is the absolute square of $F(\omega)$, for the samples in Fig. 7(a). At the commensurate field of $B / B_{\phi}=1.0, S(\omega)$ has a strict narrow band character, while at the incommensurate field of $B / B_{\phi}=$ 0.828 , the velocity noise is broad band, as indicated by the solid line which is a fit to $S(\omega) \propto \omega^{-1.75}$. There are still some weaker periodic peaks for $B / B_{\phi}=0.828$ since there is still a periodic component of the velocity induced by the substrate, and the positions of these peaks match the positions of the peaks found for $B / B_{\phi}=1.0$; however, the overall noise power

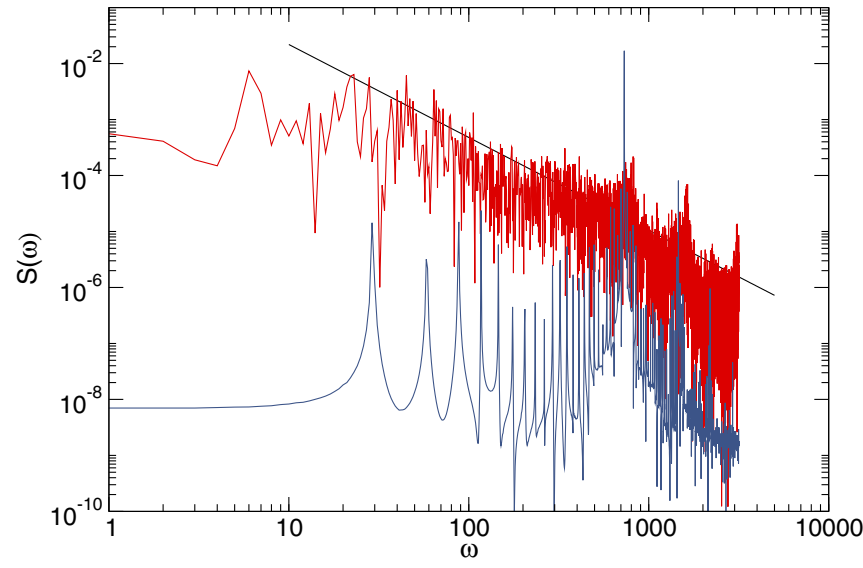

FIG. 8. (a) The power spectra $S(\omega)$ for the samples in Fig. 7(a) with $F_{p}=0.375$ and $F_{D}=1.0$ at the commensurate field $B / B_{\phi}=1.0$ (blue), where the noise is strictly narrow band, and at the incommensurate field $B / B_{\phi}=0.828$, where the noise is broad. The solid line is a fit to $S(\omega) \propto \omega^{-1.75}$.

is much larger for the incommensurate system, indicating that the driven particle velocity fluctuations extend out to much longer time scales. The presence of broad band noise or $\omega^{-\alpha}$ noise in driven systems with random disorder is associated with plastic or disordered flow, while narrow band noise is associated with ordered flow [9]. The velocity noise power spectrum for the incommensurate system with $B / B_{\phi}=1.55$ has broad band features similar to those shown for $B / B_{\phi}=$ 0.828 . Our results indicate that at the incommensurate fields, the flow has plastic characteristics since the driven particle generates position exchanges among the background vortices. Due to the periodic boundary conditions, the driven particle moves repeatedly through the sample; however, during each passage the vortex positions are rearranged by plastic events, so the landscape experienced by the driven particle changes over time, producing the large low frequency velocity noise. In contrast, under commensurate conditions the background particles are fixed and the driven particle experiences the same background during each pass through the sample. It is also possible for strictly periodic flow to occur for fillings slightly away from commensuration, $B / B_{\phi}=1.0 \pm \epsilon$, when a small number of vacancies or interstitials are present in the background lattice. As long as $\epsilon$ is small enough that the vacancies or interstitials are well spaced and immobile, the driven particle experiences a periodic potential from the background lattice at one frequency, along with lower frequency periodic perturbations from the interstitials or vacancies. When $\epsilon$ becomes too large, interactions among the interstitials or vacancies begin to occur and plastic deformations of the background lattice become possible, causing the system to transition to a broad band velocity noise regime of the type shown in Fig. 8.

In Fig. 9, we plot the velocity time series for filling fractions at and on either side of the $B / B_{\phi}=1.0$ matching field, where the velocities have been shifted vertically for clarity. The velocity fluctuations below matching for $B / B_{\phi}=$ 0.9328 are much stronger than those found above matching for $B / B_{\phi}=1.24$ and $B / B_{\phi}=1.29$. Here, the vacancy sites 


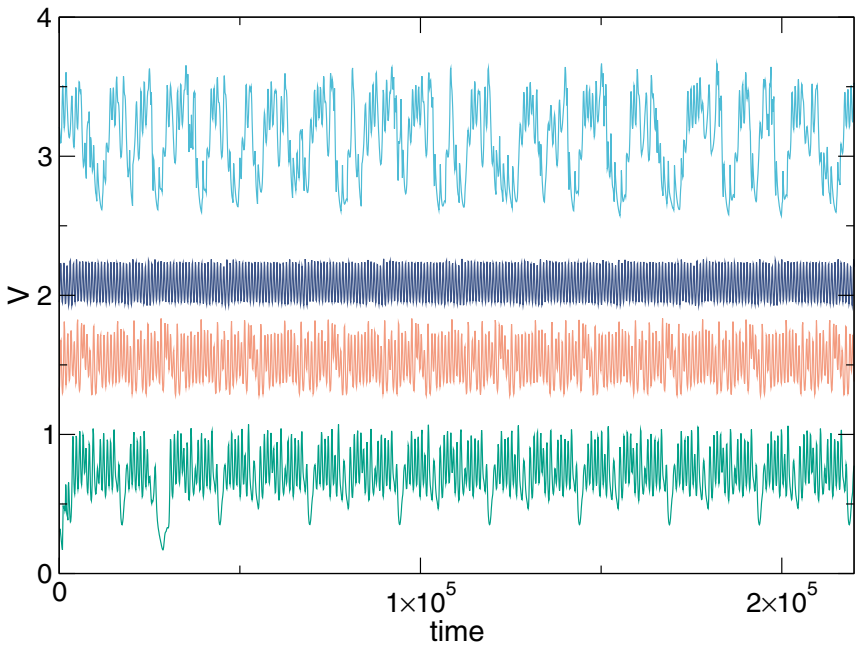

FIG. 9. (a) The time series of the driven particle velocity $V$ for the system in Fig. 7(a) with $F_{p}=0.375$ and $F_{D}=1.0$ at $B / B_{\phi}=$ 0.9328 (light blue), 1.0 (dark blue), 1.24 (orange), and 1.29 (green). The curves have been shifted vertically for clarity. The most ordered motion occurs for $B / B_{\phi}=1.0$.

which form below matching can act as trapping sites for the driven particle, permitting strong perturbations of the motion to occur. In contrast, for $B / B_{\phi}=1.24$ the average velocity is relatively high and the fluctuations are reduced in magnitude, since these fluctuations are produced by the much weaker interactions of the driven particle with interstitial vortices. For $B / B_{\phi}=1.29$, the density of interstitial vortices has become large enough that the interstitials begin to interact with each other, permitting plastic motion to occur and resulting in stronger periodic velocity drops of the driven particle.

In general, we find that for commensurate states associated with peaks in the average velocity $\langle V\rangle$, the flow of the driven particle is ordered and exhibits a periodic velocity signature, while at incommensurate fillings, the flow is plastic and has a broad band noise signal. In Figs. 10(a) and 10(b), we plot the velocity time series $V(t)$ and power spectrum $S(\omega)$ for the system in Fig. 3(a) with $F_{p}=0.375$ and $F_{D}=1.0$ at $B / B_{\phi}=3.0$. For this filling, there is no peak in $\langle V\rangle$ and the background vortices do not form an ordered square or triangular lattice but instead adopt a disordered structure. As the probe particle passes through the sample, it generates strong plastic deformations of the background vortices, and the velocity noise has a broad band character. For $B / B_{\phi}=4.03$ in Figs. 10(c) and $10(\mathrm{~d})$, slightly above the fourth matching field where a peak in $\langle V\rangle$ appears in Fig. 3(a), the background vortices form a triangular lattice that contains a few grain boundaries. Here, the driven particle spends most of its time traversing ordered portions of the lattice and exhibits a dip in $V$ each time it crosses one of the grain boundaries. The power spectrum indicates that two separate periodicities are combined in the velocity signal. The higher frequency peaks are associated with the motion of the driven particle through the periodic commensurate vortex lattice, while the lower frequency narrow band noise is produced by the periodic motion of the probe particle over the grain boundaries. A given grain boundary can move gradually over time since it can be entrained

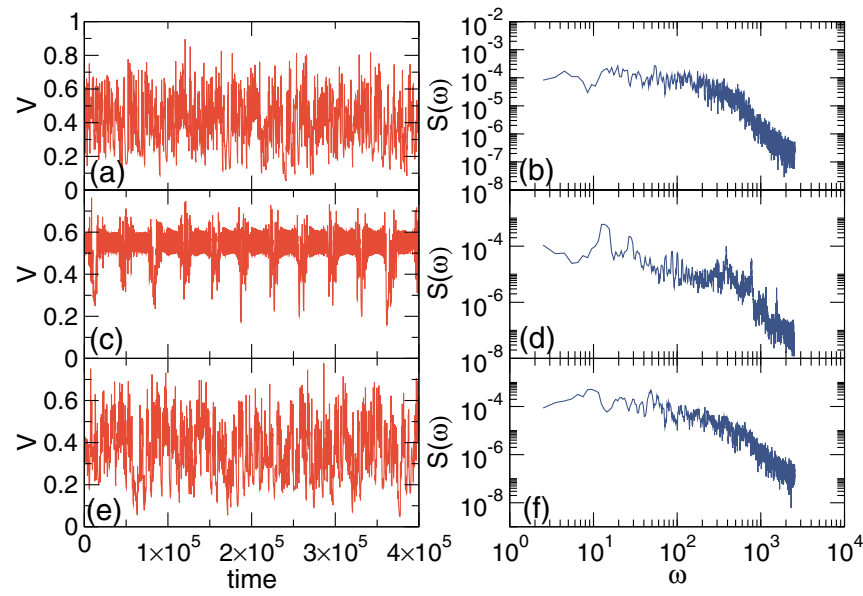

FIG. 10. [(a), (c), and (e)] The velocity time series $V(t)$ and [(b), (d), and (f)] the corresponding power spectra $S(\omega)$ for the system in Fig. 3(a) with $F_{p}=0.375$ and $F_{D}=1.0$. [(a) and (b)] $B / B_{\phi}=3.0$, where the system is disordered. [(c) and (d)] $B / B_{\phi}=4.03$, where the system is ordered. [(e) and (f)] $B / B_{\phi}=4.4576$, where the vortex positions are more disordered and the velocity power spectrum has a broad band character.

briefly by the probe particle, producing a drop in the probe particle velocity. At $B / B_{\phi}=4.4576$ in Figs. 10 (e) and $10(\mathrm{f})$, the vortex positions are more disordered and the velocity time series has chaotic features, while the power spectrum has a broad band noise shape.

In Fig. 11(a), we show the bulk vortices, pinning sites, driven vortex, and vortex trajectories for a portion of a system with $F_{p}=0.375$ and $F_{D}=1.0$ at $B / B_{\phi}=1.55$. The background vortices are disordered and the trajectory of the probe particle is also disordered, giving the strongly fluctuating velocity signature shown in Fig. 7(b) and producing broad band velocity noise. At $B / B_{\phi}=2.0$, where there is a peak in $\langle V\rangle$ and the background vortices form an ordered square lattice, Fig. 11(b) indicates that the driven particle moves through the interstitial region along a sinuous path modulated by the interstitial vortices, resulting in a narrow band noise signature. In Fig. 11(c) at $B / B_{\phi}=3.0$, the background vortices are not crystalline, consistent with the absence of a peak in $\langle V\rangle$ at this filling. The driven vortex follows a disordered path and exhibits broad band noise. Figure 11(d) shows that for $B / B_{\phi}=4.03$, the background vortices form a polycrystalline lattice and the flow is mostly ordered, giving the narrow band noise illustrated in Figs. 10(c) and 10(d).

Based on the features in $\langle V\rangle$ and $S(\omega)$, we construct a dynamic phase diagram as a function of pinning strength $F_{p}$ versus $B / B_{\phi}$ with $F_{D}=1.0$ in Fig. 12 , where we highlight the pinned, viscous flow or broad band noise, and narrow band noise regimes. Only the largest pinned phases are marked; there are several smaller pinned phases which are not shown on the figure. For finite $F_{p}$, at low fillings the system is always in a periodic motion state since the driven particle moves along the pinning rows as shown in Fig. 5(a). Similar periodic oscillations arise around $B / B_{\phi}=1 / 2,1.0,2.0$, and 4.0. The pinned regions reach their greatest extent for incommensurate fillings, consistent with our observation that the effectiveness of the pinning is generally reduced at commensurate fillings. 

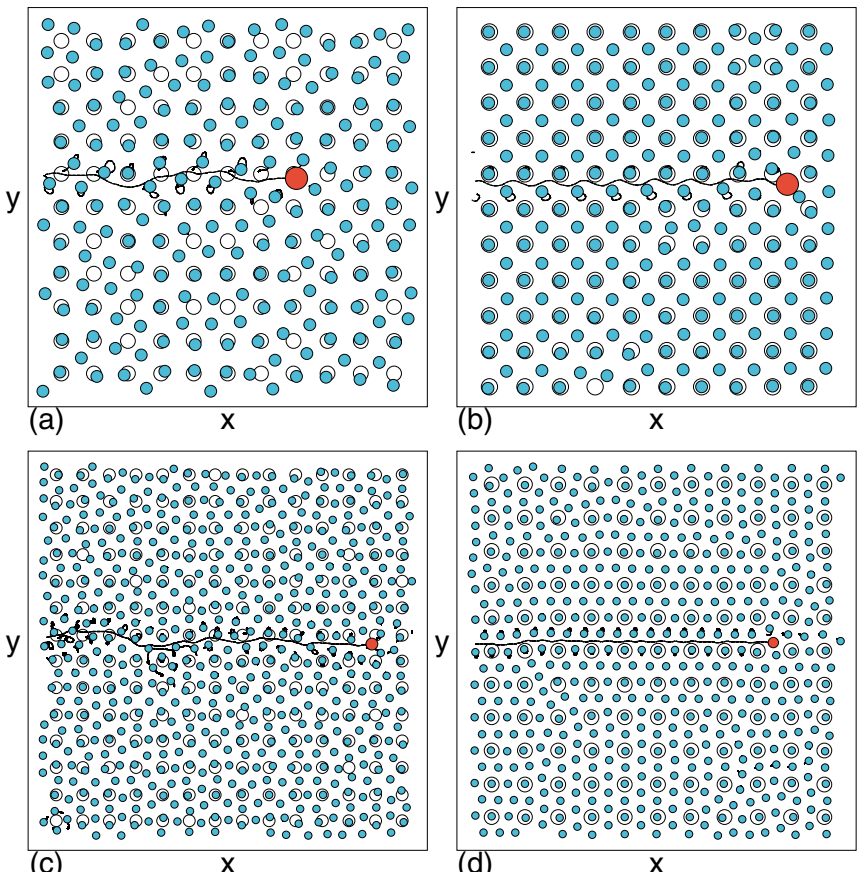

(b)

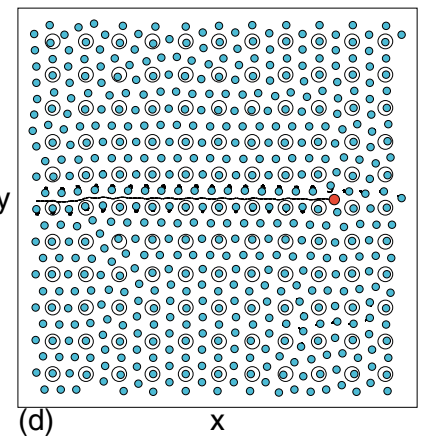

(d)

FIG. 11. Image of a subsection of the superconducting vortex system with a square pinning array (open circles), bulk vortices (blue circles), the driven vortex (red circle), and the vortex trajectories for a system with $F_{p}=0.375$ and $F_{D}=1.0$. The size of the pinning sites has been adjusted for clarity. (a) Disordered flow at $B / B_{\phi}=1.55$. (b) Ordered flow at $B / B_{\phi}=2.0$. (c) Disordered flow at $B / B_{\phi}=3.0$. (d) Ordered flow at $B / B_{\phi}=4.03$.

For low fillings, the driven particle does not become pinned until $F_{D} \geqslant F_{p}$. For $B / B_{\phi}>3.0$, there are windows of $B / B_{\phi}$ over which the driven particle never becomes pinned, since all of the pinning sites are occupied and the driven particle can only be pinned through caging by interactions with neigh-

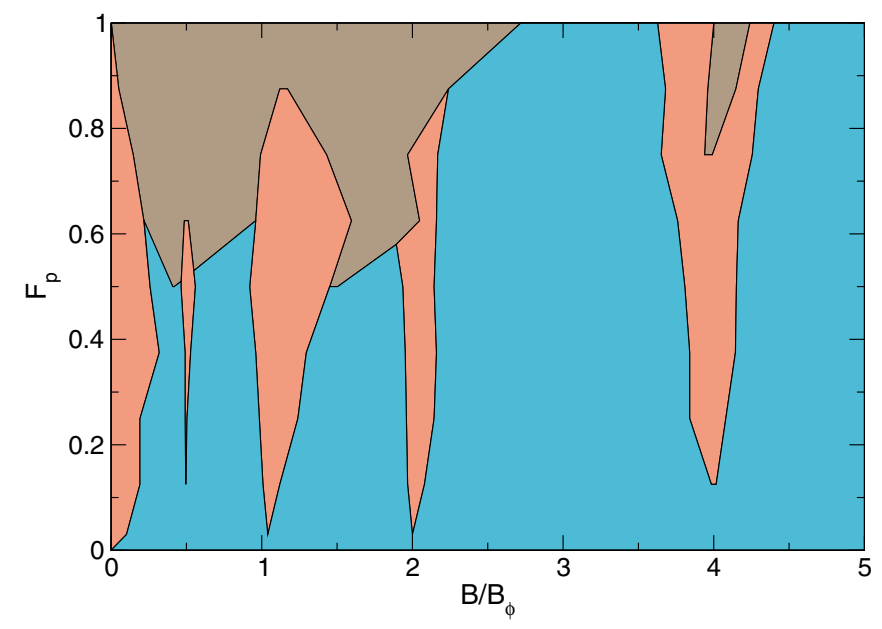

FIG. 12. (a) Dynamic phase diagram as a function of pinning strength $F_{p}$ vs filling $B / B_{\phi}$ highlighting the pinned phase (brown), the viscous flow or broad band noise phase (blue), and the periodic flow or narrow band noise phase (orange) for the system from Figs. 3 and 4 with $F_{D}=1.0$. Only the largest pinned regions are marked; smaller pinned regimes are not shown.

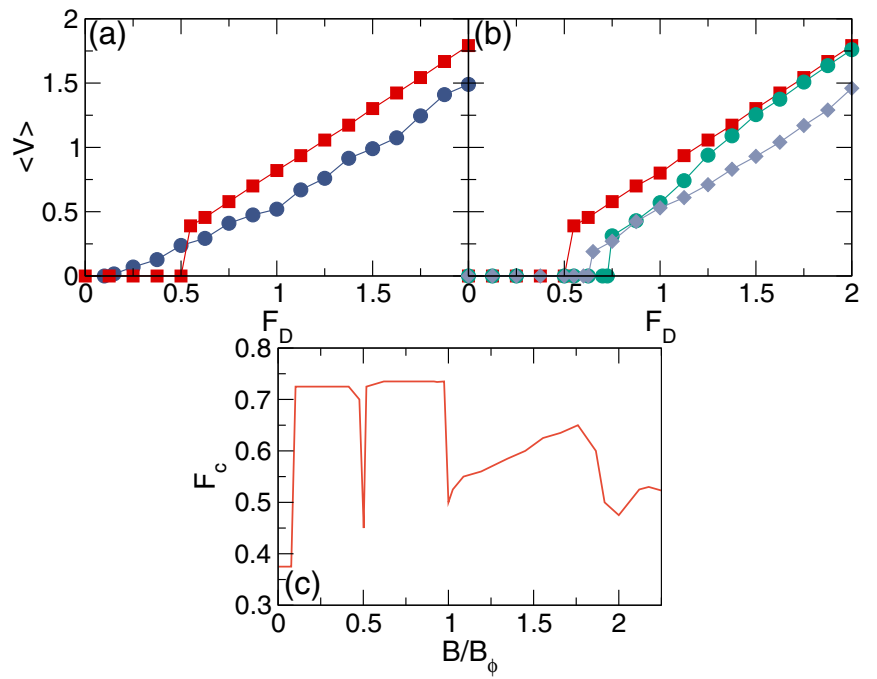

FIG. 13. (a) $\langle V\rangle$ vs driving force $F_{D}$ for the system in Fig. 3 at $B / B_{\phi}=1.0$ with $F_{p}=0.0$ (blue circles) and $F_{p}=0.375$ (red squares). (b) $\langle V\rangle$ vs $F_{D}$ for the same system at $F_{p}=0.375$ for $B / B_{\phi}=0.9328$ (green circles), 1.0 (red squares), and 1.55 (light blue diamonds). (c) The critical depinning force $F_{c}$ vs $B / B_{\phi}$ for the same system.

boring vortices. At lower pinning strengths, the flow is not periodic even at the matching fields since as the driven particle moves it can interact strongly enough with the background vortices to generate plastic flow events.

We next consider the drive dependence and pinning transition for the driven particle. In Fig. 13(a), we plot the velocity-force curve $\langle V\rangle$ versus $F_{D}$ of the driven vortex for the system in Fig. 3 at $B / B_{\phi}=1.0$ for $F_{p}=0.0$ and $F_{p}=0.375$. In the sample with no pinning, there is still a finite depinning transition just above $F_{D}=0.1$ produced by the caging of the driven vortex due to its interactions with the surrounding vortex lattice. When $F_{p}=0.375$, the depinning transition occurs at $F_{p}=0.5$, about five times higher than in the sample without pinning, and is much sharper. In the pin-free sample, the depinning transition is plastic and depinning occurs when vortices in the surrounding lattice become able to exchange places with each other. For the finite pinning sample, the depinning is elastic and the driven particle moves without creating any plastic distortions in the surrounding lattice. This motion can be viewed as a single particle traveling over a fixed square $2 \mathrm{D}$ substrate. Once the particle is moving, $\langle V\rangle$ is higher for the sample containing pinning than for the sample without pinning. The pinning prevents the surrounding vortices from being dragged by the driven particle or from exchanging places with each other in plastic events, both of which are processes which increase the drag on the driven particle. This is consistent with the results in Fig. 2 where $\langle V\rangle$ is lower for a sample with finite pinning than for a sample with no pinning.

In Fig. 13(b), we plot $\langle V\rangle$ versus $F_{D}$ for the system with $F_{p}=0.375$ at $B / B_{\phi}=0.9328,1.0$, and 1.55 . The depinning threshold is lowest when $B / B_{\phi}=1.0$, and in the flowing phase, $\langle V\rangle$ is smaller at incommensurate fillings than at the commensurate filling. Figure 13(c) shows the critical depinning force $F_{c}$ versus $B / B_{\phi}$ obtained from a series of 


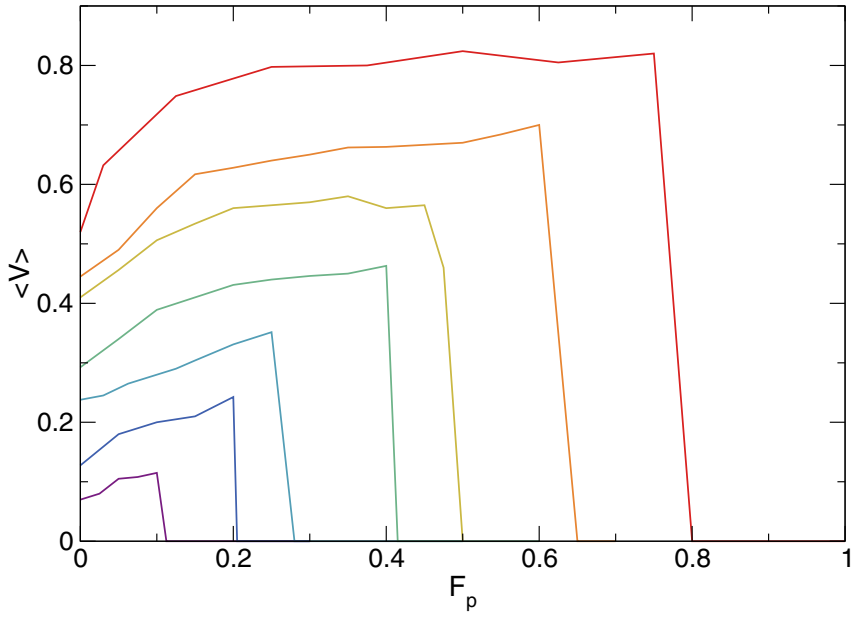

FIG. 14. $\langle V\rangle$ vs $F_{p}$ at $B / B_{\phi}=1.0$ for the system in Fig. 3 at $F_{D}=$ $1.0,0.825,0.75,0.625,0.5,0.375$, and 0.25 , from top to bottom, showing that for fixed drive, there is a regime in which $\langle V\rangle$ increases with increasing $F_{p}$.

velocity-force curves for the system in Fig. 13(b). For $B / B_{\phi}<$ 0.075 , we find $F_{c}=F_{p}$ since the depinning occurs in the single particle limit and is controlled only by direct depinning from the pinning sites, with vortex-vortex interactions playing no role. For $0.075 \leqslant B / B_{\phi}<1 / 2$, the depinning threshold reaches its largest value of $F_{c}=0.725$, produced by a combination of direct pinning effects and additional interstitial pinning due to interactions with the neighboring vortices. At $B / B_{\phi}=1 / 2$, where the system forms a checkerboard ordered state, the driven particle moves along a 1D path between the pinning sites and the only pinning arises from interactions with other vortices. Here the driven particle does not move directly into a pinned vortex; instead, it flows around the side of the pinned vortex where it experiences a smaller repulsive force. For $1 / 2<B / B_{\phi}<1.0$, the system is disordered and the driven particle experiences pinning from both the pinning sites and the pinned vortices. The depinning force drops again at $B / B_{\phi}=1.0$ when the driven particle follows a $1 \mathrm{D}$ path between the pinning sites, as illustrated in Fig. 1. For $B / B_{\phi}>1.0$, most of the pinning sites are occupied so $F_{c}$ is generally lower. There is another drop in $F_{c}$ at $B / B_{\phi}=2.0$ where the driven particle traverses a quasi-1D path between the pinning rows, as shown in Fig. 11(b), while for $B / B_{\phi}>$ 2.0, $F_{c}$ increases again. This result indicates that the depinning threshold for the single driven vortex is lowest at matching fields where there is an ordered configuration of background vortices, which is opposite to the behavior found for bulk driven vortices.

For constant $F_{D}$ within the moving phase, the velocity response increases with increasing $F_{p}$, opposite to what is observed in most systems with increasing pinning force [9]. In Fig. 14, we plot $\langle V\rangle$ versus $F_{p}$ at $B / B_{\phi}=1.0$ for the system in Fig. 3 at $F_{D}=1.0,0.825,0.75,0.625,0.5,0.375$, and 0.25. For a fixed driving force, the velocity is low at $F_{p}=0$ and increases with increasing $F_{p}$ before reaching a saturation and then dropping to zero when $F_{p}$ becomes large enough. For all the curves, when $F_{p}<0.06$ the system is in a viscous or plastic flow regime since the pinning is no longer strong
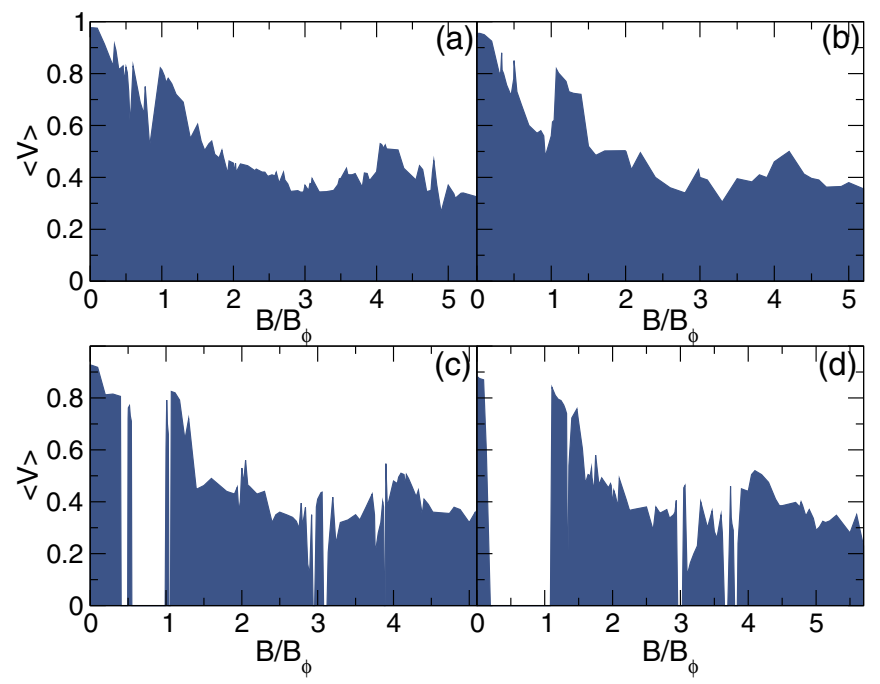

FIG. 15. (a) $\langle V\rangle$ vs $B / B_{\phi}$ for a system with a triangular pinning array at $F_{D}=1.0$ and $F_{p}=$ (a) 0.375 , (b) 0.5 , (c) 0.625 , and (d) 0.75 . A series of peaks in $\langle V\rangle$ appear at matching fields for which the system forms an ordered lattice.

enough to hold the background vortices in place. At higher $F_{p}$, the background vortices become pinned and the system enters a periodic channel flow regime.

\section{TRIANGULAR PINNING}

We next consider the average velocity $\langle V\rangle$ of a single particle driven through a background of vortices interacting with a triangular pinning array under constant drive. In Fig. 15, we plot $\langle V\rangle$ versus $B / B_{\phi}$ for a system with a triangular pinning array at $F_{D}=1.0$ and $F_{p}=0.375,0.5,0.625$, and 0.75 . At $F_{p}=$ 0.375 in Fig. 15(a), there are large peaks in $\langle V\rangle$ at $B / B_{\phi}=1.0$ and 4.0 along with smaller peaks at $B / B_{\phi}=1 / 3$ and 3.0. There is no peak at $B / B_{\phi}=1 / 2$ since for this filling the background vortices form a polycrystalline disordered state. For $F_{p}=0.5$ in Fig. 15(b), we observe the same trend, but the peak at $B / B_{\phi}=3.0$ is more prominent since the system can achieve better ordering at this field when the pinning is stronger. Additionally, a peak begins to emerge at $B / B_{\phi}=1 / 2$ due to the formation of a stripe-like pattern. From previous studies of vortex ordering on triangular arrays, it is known that an ordered state can occur at $B / B_{\phi}=1 / 3$ and a partially ordered state can form at $B / B_{\phi}=1 / 2$ [24]. At $B / B_{\phi}=3.0$ and 4.0, the background vortices form a triangular lattice, but the orientation of this lattice at $B / B_{\phi}=3.0$ involves the appearance of a zigzag pattern in the interstitial region [17] which makes it more difficult for the driven particle to pass through the sample. In contrast, at $B / B_{\phi}=4.0$ the triangular lattice is oriented such that there are $1 \mathrm{D}$ chains of interstitial vortices along the $x$ direction [17], making it possible for the driven particle to slide easily alongside the chain without weaving into the $y$ direction. At $B / B_{\phi}=5.0$, the background vortex positions are disordered and there is no peak in $\langle V\rangle$. Previous work showed that a triangular lattice oriented similarly to the lattice observed at $B / B_{\phi}=4.0$ appears at $B / B_{\phi}=9.0$ but that there are two rows of interstitial vortices instead of only one [42], so we expect that there would be another peak in $\langle V\rangle$ 

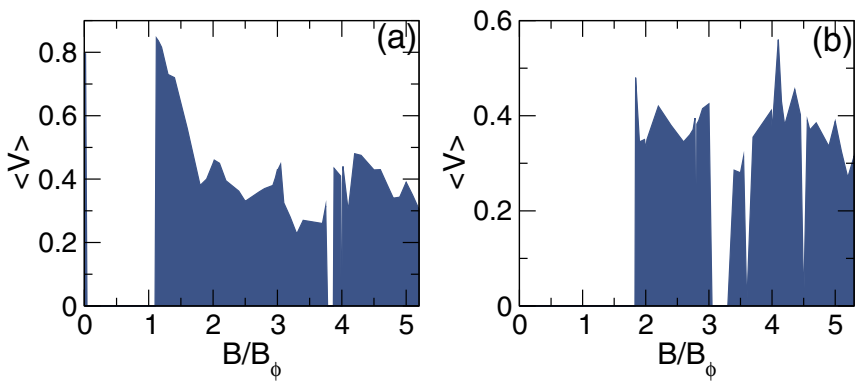

FIG. 16. (a) $\langle V\rangle$ vs $B / B_{\phi}$ for a system with a triangular pinning array at $F_{D}=1.0$ and $F_{p}=$ (a) 0.875 and (b) 1.0.

at this higher filling. For $F_{p}=0.625$ in Fig. 15(c), there are some regions such as $1 / 2<B / B_{\phi}<1.0$. where the system is pinned and $\langle V\rangle=0$. A smaller velocity peak appears at $B / B_{\phi}=2.0$ where the background vortices attempt to form an ordered honeycomb state [17], while the peaks in $\langle V\rangle$ remain robust at $B / B_{\phi}=3.0$ and 4.0. At $F_{p}=0.75$ in Fig. 15(d), an unpinned region persists for the lowest values of $B / B_{\phi}$, while the pinned region now extends above $B / B_{\phi}=1.0$. For higher pinning forces, the number and extent of pinned regions grows. We illustrate this in Fig. 16 where we plot $\langle V\rangle$ versus $B / B_{\phi}$ for samples with $F_{p}=0.875$ and $F_{p}=1.0$. In each case, the peaks in $\langle V\rangle$ near $B / B_{\phi}=3.0$ and 4.0 remain present.

In Fig. 17(a), we show the vortex positions and trajectories for the system in Fig. 15(a) with $F_{p}=0.375, F_{D}=1.0$, and $B / B_{\phi}=1.0$. For this filling, the background vortices form a commensurate triangular lattice and the driven particle moves in a sinusoidal fashion through the interstitial region. At $B / B_{\phi}=1.5$ in Fig. 17(b), the system is in a disordered state and the driven particle follows a much more random path. We note that the velocity noise for the probe particle on a triangular pinning array has features similar to those described above for the square pinning array. Under commensurate conditions, there is a peak in $\langle V\rangle$, the motion of the probe particle is periodic, and the velocity noise has a narrow band character, while for incommensurate states, the vortex configurations are

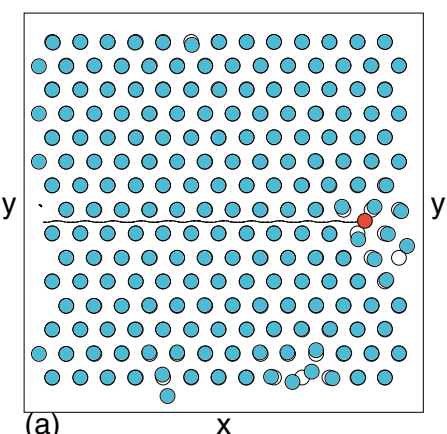

(a)

FIG. 17. Image of a subsection of the superconducting vortex system with a triangular pinning array (open circles), bulk vortices (blue circles), the driven vortex (red circle), and the vortex trajectories for the system in Fig. 15(a) with $F_{p}=0.375$ and $F_{D}=1.0$. The size of the pinning sites has been adjusted for clarity. (a) Ordered flow at $B / B_{\phi}=1.0$. (b) Disordered flow at $B / B_{\phi}=1.5$.
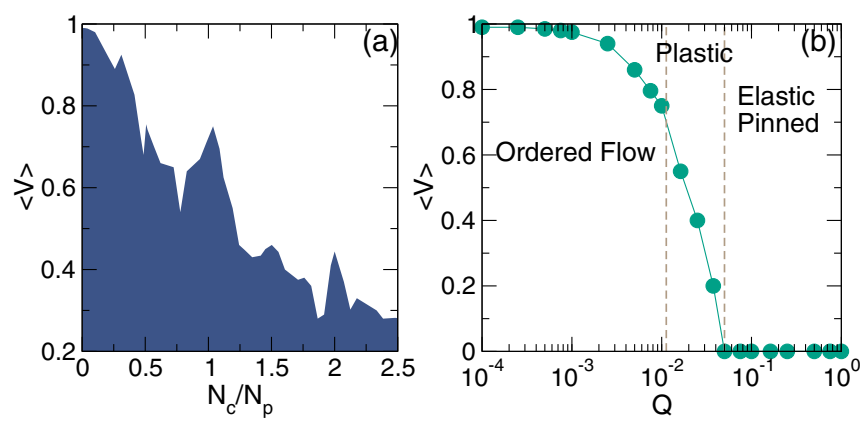

FIG. 18. (a) $\langle V\rangle$ vs $N_{c} / N_{p}$ for a driven probe particle moving through a background of colloidal particles interacting with a square pinning array, where $N_{c}$ is the number of colloids and $N_{p}$ is the number of pinning sites, for a system with colloidal charge $Q=0.01$, $F_{p}=0.25$, and $F_{D}=1.0$. (b) $\langle V\rangle$ versus colloidal charge $Q$ for the same system at $N_{c} / N_{p}=1.0, F_{p}=0.25$, and $F_{D}=1.0$. At low $Q$, the driven particle is able to move without generating distortions in the background lattice in the ordered flow state. At high $Q$, the system is elastic and forms a triangular solid as illustrated in Fig. 19(a), and the driven particle is pinned. For intermediate $Q$, the system forms a partially commensurate state of the type shown in Fig. 19(b).

disordered, there is no peak in $\langle V\rangle$, and the velocity noise is broad band.

\section{COLLOIDAL PARTICLES}

We next consider the case of a driven colloidal probe particle moving through a background colloidal lattice that is interacting with a square pinning array. Here the Bessel function vortex-vortex interaction is replaced with a screened Yukawa colloid-colloid interaction potential of the form $U(r)=Q \exp (-\kappa r) / r$. In Fig. 18, we plot $\langle V\rangle$ versus $N_{c} / N_{p}$, the ratio of the number of colloids $N_{c}$ to the number of pinning sites $N_{p}$, for a system with a colloidal charge of $Q=0.01, F_{p}=0.25$, and $F_{D}=1.0$. Peaks in $\langle V\rangle$ appear at fillings $N_{c} / N_{p}=1.0$ and 2.0, similar to what is observed in the superconducting vortex system.

The charge on the colloidal particles can be varied, making it possible to hold the colloid density fixed while passing from the strongly charged limit, in which the background colloids form a stiff triangular lattice that floats above the substrate, to the weakly charged limit, in which the pinning site locations dominate the behavior. In most bulk driven systems with pinning, increasing the interactions between the particles causes the depinning threshold to drop, and a transition can occur from plastic depinning, where a portion of the particles are trapped by pinning sites while the remaining particles begin to move, to elastic depinning, where all of the particles move simultaneously and maintain the same neighbors [9]. In general, the elastic depinning threshold is much lower than the plastic depinning threshold. For a single driven probe particle, we find the opposite behavior, in which the driven particle becomes pinned when the background colloid interactions become strong enough for elastic behavior to emerge.

In Fig. 18(b), we plot $\langle V\rangle$ versus $Q$ for the system in Fig. 18(a) at $N_{c} / N_{p}=1.0, F_{p}=0.25$, and $F_{D}=1.0$. For $Q>$ 0.05 , the system forms a floating triangular solid of the type 


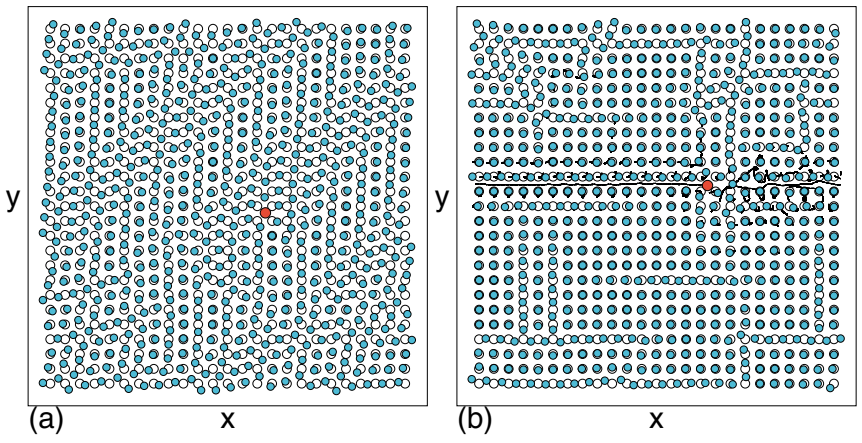

FIG. 19. Image of a subsection of the colloidal system with a square pinning array (open circles), bulk vortices (blue circles), the driven vortex (red circle), and the vortex trajectories for the system in Fig. 18 with $F_{p}=0.25$, and $F_{D}=1.0$. The size of the pinning sites has been adjusted for clarity. (a) The pinned phase at $Q=0.1$ where the system forms a floating triangular solid. (b) The partially commensurate phase at $Q=0.0375$ where the flow is plastic.

illustrated in Fig. 19(a) at $Q=0.1$. Here the interactions between the colloidal particles are so strong that the elastic energy of the colloidal lattice overcomes the pinning energy of the substrate, and the background colloids float above the substrate potential instead of sitting in the pinning sites. Under these conditions, the driven particle cannot tear plastically past the background colloids and must instead push the entire system as a unit, so $\langle V\rangle$ is close to zero. For $0.01<Q<0.05$, the pinning energy begins to play a role and the background colloids form a partially commensurate or polycrystalline solid in which portions of the colloids are commensurate with the underlying pinning substrate while other portions are not. The driven particle depins plastically, generating distortions in the background colloids as illustrated in Fig. 19(b) for $Q=0.0375$. The plastic deformations produce a large drag on the driven particle, giving a reduced $\langle V\rangle$. At $Q=0.01$, the background colloidal particles form a commensurate solid similar to that shown in Fig. 1, and the driven particle flows along a $1 \mathrm{D}$ channel without producing any plastic distortion in the lattice. As $Q$ is decreased further, $\langle V\rangle$ continues to increase since the drag on the driven particle diminishes, and the system enters a weakly interacting limit in which the flow is along a strictly $1 \mathrm{D}$ channel. At even lower $Q$, each pinning site can begin to trap multiple particles; however, since $F_{D}>F_{p}$, the driven particle will continue to flow even if it occasionally passes through an empty pinning site.

\section{DISCUSSION}

There are some limited examples in the literature of commensurate states that produce peaks instead of dips in the transport velocity at commensurate fillings. In the work of Poccia et al. [73] on superconducting vortices moving over a square pinning array, at matching fields the differential resistance showed dips at low current, indicating enhanced pinning or reduced vortex velocities, but at higher currents these dips became peaks, indicating that the vortices are moving faster at the commensurate fillings than at the incommensurate fields. Poccia et al. described these results in terms of a dynamic Mott transition. It is possible that for low currents, the vortex motion is dominated by the commensurate vortices trapped at the pinning sites, but for higher currents, there could be additional interstitial vortices induced by the current, and these additional vortices could flow through the ordered pinned vortices in a manner similar to the motion shown in Fig. 1. At incommensurate fields, there are still additional current-induced vortices when higher currents are applied, but the vortex lattice is already disordered so the additional drag induced by these extra vortices is not noticeable. Jiang et al. also found a similar transition from dips to peaks in the differential resistance of $\mathrm{Nb}$ films with periodic pinning arrays as a function of increasing drive [74]. A key difference between these experimental results and what we observe in our simulations is that the velocity peaks we find occur even for low drives, indicating that the system is always behaving in an anticommensurate fashion, whereas in Refs. [73,74], the velocity is enhanced at matching conditions only for high drives.

Regarding classification of commensurate and incommensurate states, in Ref. [1] a commensurate structure is defined to occur when the average lattice spacing $a$ is a simple rational fraction of the substrate period $b$. Depending on the strength of the substrate, the system may pass through an infinite number of commensurate states without locking to any of them, or it may lock to certain commensurate states; in addition, there may or may not be incommensurate states between the commensurate states. One may try to use a simple criterion such as a relation between lattice unit cell vectors of the vortices and the pinning substrate to decide whether a commensurate state is expected to appear; however, this neglects the complex grain boundary and dislocation structures that can arise in the actual system at certain fillings. Examples of such structures can be found for commensurate states in Ref. [42] and for incommensurate states in Ref. [6]. A full treatment of the complexity of the commensuration behavior is outside the scope of the present work but is an interesting direction for future study.

In our work, we always drive along a symmetry direction of the pinning array; however, the results could change for driving along other directions. In the case of the square pinning array, driving along the positive or negative $y$ direction should produce the same effects as driving along the $x$ direction due to the lattice symmetry. We also expect similar effects to appear for driving along other angles at which it is possible for the driven particle to follow a 1D path without encountering a pinning site, such as for driving at $45^{\circ}$ from the $x$ direction. In previous work, it was shown that particles move more easily along what are known as commensurate angles on a square lattice given by $\theta=\arctan (n / m)$ with integer $m$ and $n[75,76]$, so if the driving of the individual particle is aligned with an incommensurate angle, the behavior might be more similar to what is observed for driving an individual particle over random disorder.

In our model, the pinning is represented as localized sites which can capture at most one particle. Other types of pinning arrays also exist, such as egg carton potentials which have no interstitial regions $[18,19]$. For such a potential, at higher fillings including $f=2$, each potential minimum could capture two particles which would form a dimer like state. We expect that anticommensuration effects would still occur in 
these potentials, since at matching conditions the background particles would be more strongly coupled to the substrate than to the driven particle, just as in the case of our localized pinning sites.

Our results should be general to the broader class of systems that exhibit commensurability effects and that include an individually driven particle. Our results are also relevant for understanding the dynamics near commensurate states in the presence of some form of doping, such as an additional particle with different properties or a different size compared to the other particles. Under incommensurate conditions, the drag on these doping particles would be increased, but the drag would be reduced in and near commensurate states. Similar effects could occur in systems containing multiple species that interact with each other and with a periodic substrate. If one species becomes more strongly coupled to the substrate, such as at matching conditions, the drag on the other species could be reduced. Such behavior could be relevant for multicomponent Bose-Einstein condensates or colloidal systems with multiple species.

\section{SUMMARY}

We have examined the active rheology for a driven particle moving through an assembly of other particles in the presence of a periodic pinning substrate. Under bulk driving, it was previously observed that a series of commensuration effects in the form of a peak in the depinning force or a dip in the velocity occur when the number of particles is an integer or rational fractional multiple of the number of pinning sites. In this active rheology study, where only a single particle is driven, we measure the velocity of the driven particle at a fixed driving force and varied filling factors for both vortices in type-II superconductors and colloidal particles interacting with periodic pinning arrays. We find an interesting anticommensuration effect in which the velocity of the driven particle exhibits peaks instead of dips at the commensurate configurations, a behavior which is the opposite of what is observed in bulk driven systems. Under nonmatching conditions, the system forms a disordered state in which the driven particle entrains a greater number of background particles and/or experiences a greater number of collisions with pinning sites. We show that peaks in the velocity only occur at matching fields for which the system forms an ordered lattice. Under these conditions, the background particles form an ordered lattice which is more strongly coupled to the substrate than to the driven particle, causing a reduction in the drag on the driven particle. The probe particle motion at commensurate conditions is ordered and the velocity has a narrow band noise signature, while at incommensurate fillings, the motion is disordered and the noise signature becomes broad band. In general, the probe particle has a larger velocity in the presence of pinning than in the absence of pinning regardless of whether the filling is commensurate or incommensurate. The velocity-force curves indicate that at commensuration, the depinning threshold is reduced and the velocity in the moving phase is enhanced. In some cases, we also find dips in the probe particle velocity and depinning force at fractional fillings such as $f=1 / 2$. Within the moving phase, the velocity of the probe particle under constant driving increases with increasing pinning force, a behavior opposite from what is found in bulk driven systems. We show that these effects are robust for both square and triangular pinning arrays. We also find similar effects for colloidal particles interacting with a square substrate for varied filling factors. In the case of colloidal particles, when the colloidcolloid interactions are strengthened by increasing the charge on each colloid, the background colloidal particles form a triangular lattice which floats above the pinning substrate, and the probe particle becomes pinned within this lattice, while for lower charges and weaker colloid-colloid interactions, the probe particle can depin and the system enters either a plastic flow or ordered flow regime. This is the opposite of what is observed in bulk driven systems, where weak particle-particle interactions result in strong coupling to the substrate and an enhanced pinning effect, while strong particle-particle interactions produce a highly elastic particle lattice with weak coupling to the substrate and a reduced pinning effect. Our results should be general to a range of systems in which particles are coupled to periodic substrates. The anticommensuration effects may also explain recent observations of vortex motion in periodic pinning arrays, where there is a transition from dips to peaks in the vortex velocity at matching fields as the applied drive is increased. Our results could also be relevant to certain systems of multiple species coupled to a substrate in which the drag on one species is reduced at commensurate conditions when one species couples strongly to the substrate while the other species does not.

\section{ACKNOWLEDGMENTS}

We gratefully acknowledge the support of the U.S. Department of Energy through the LANL/LDRD program for this work. This work was supported by the US Department of Energy through the Los Alamos National Laboratory. Los Alamos National Laboratory is operated by Triad National Security, LLC, for the National Nuclear Security Administration of the U. S. Department of Energy (Contract No. 892333218NCA000001).
[1] P. Bak, Commensurate phases, incommensurate phases and the devil's staricase, Rep. Prog. Phys. 45, 587 (1982).

[2] K. Harada, O. Kamimura, H. Kasai, T. Matsuda, A. Tonomura, and V. V. Moshchalkov, Direct observation of vortex dynamics in superconducting films with regular arrays of defects, Science 274, 1167 (1996).

[3] J. Hu and R. M. Westervelt, Commensurate-incommensurate transitions in magnetic bubble arrays with periodic line pinning, Phys. Rev. B 55, 771 (1997).
[4] K. Mangold, P. Leiderer, and C. Bechinger, Phase Transitions of Colloidal Monolayers in Periodic Pinning Arrays, Phys. Rev. Lett. 90, 158302 (2003).

[5] S. Tung, V. Schweikhard, and E. A. Cornell, Observation of Vortex Pinning in Bose-Einstein Condensates, Phys. Rev. Lett. 97, 240402 (2006).

[6] D. McDermott, J. Amelang, L. M. Lopatina, C. J. Olson Reichhardt, and C. Reichhardt, Domain and stripe formation between hexagonal and square ordered fillings of colloidal 
particles on periodic pinning substrates, Soft Matter 9, 4607 (2013).

[7] A. Vanossi, N. Manini, M. Urbakh, S. Zapperi, and E. Tosatti, Colloquium: Modeling friction: From nanoscale to mesoscale, Rev. Mod. Phys. 85, 529 (2013).

[8] T. Bohlein, J. Mikhael, and C. Bechinger, Observation of kinks and antikinks in colloidal monolayers driven across ordered surfaces, Nat. Mater. 11, 126 (2012).

[9] C. Reichhardt and C. J. Olson Reichhardt, Depinning and nonequilibrium dynamic phases of particle assemblies driven over random and ordered substrates: A review, Rep. Prog. Phys. 80, 026501 (2017).

[10] J. Gutierrez, A. V. Silhanek, J. Van de Vondel, W. Gillijns, and V. V. Moshchalkov, Transition from turbulent to nearly laminar vortex flow in superconductors with periodic pinning, Phys. Rev. B 80, 140514(R) (2009).

[11] S. Avci, Z. L. Xiao, J. Hua, A. Imre, R. Divan, J. Pearson, U. Welp, W. K. Kwok, and G. W. Crabtree, Matching effect and dynamic phases of vortex matter in $\mathrm{Bi}_{2} \mathrm{Sr}_{2} \mathrm{CaCu}_{2} \mathrm{O}_{8}$ nanoribbon with a periodic array of holes, Appl. Phys. Lett. 97, 042511 (2010).

[12] A. Vanossi, N. Manini, and E. Tosatti, Static and dynamic friction in sliding colloidal monolayers, Proc. Natl. Acad. Sci. (USA) 109, 16429 (2012).

[13] O. Daldini, P. Martinoli, J. L. Olsen, and G. Berner, Vortex-Line Pinning by Thickness Modulation of Superconducting Films, Phys. Rev. Lett. 32, 218 (1974).

[14] O. V. Dobrovolskiy, E. Begun, M. Huth, and V. A. Shklovskij, Electrical transport and pinning properties of $\mathrm{Nb}$ thin films patterned with focused ion beam-milled washboard nanostructures, New J. Phys. 14, 113027 (2012).

[15] Q. Le Thien, D. McDermott, C. J. Olson Reichhardt, and C. Reichhardt, Orientational ordering, buckling, and dynamic transitions for vortices interacting with a periodic quasi-onedimensional substrate, Phys. Rev. B 93, 014504 (2016).

[16] M. Baert, V. V. Metlushko, R. Jonckheere, V. V. Moshchalkov, and Y. Bruynseraede, Composite Flux-Line Lattices Stabilized in Superconducting Films by a Regular Array of Artificial Defects, Phys. Rev. Lett. 74, 3269 (1995).

[17] C. Reichhardt, C. J. Olson, and F. Nori, Commensurate and incommensurate vortex states in superconductors with periodic pinning arrays, Phys. Rev. B 57, 7937 (1998).

[18] C. Reichhardt and C. J. Olson, Colloidal dynamics on Disordered Substrates, Phys. Rev. Lett. 89, 078301 (2002).

[19] M. Brunner and C. Bechinger, Phase Behavior of Colloidal Molecular Crystals on Triangular Light Lattices, Phys. Rev. Lett. 88, 248302 (2002).

[20] J. I. Martín, M. Vélez, J. Nogués, and I. K. Schuller, Flux Pinning in a Superconductor by an Array of Submicrometer Magnetic Dots, Phys. Rev. Lett. 79, 1929 (1997).

[21] A. N. Grigorenko, S. J. Bending, M. J. Van Bael, M. Lange, V. V. Moshchalkov, H. Fangohr, and P. A. J. de Groot, Symmetry Locking and Commensurate Vortex Domain Formation in Periodic Pinning Arrays, Phys. Rev. Lett. 90, 237001 (2003).

[22] G. R. Berdiyorov, M. V. Milošević, and F. M. Peeters, Novel Commensurability Effects in Superconducting Films with Antidot Arrays, Phys. Rev. Lett. 96, 207001 (2006).

[23] M. L. Latimer, G. R. Berdiyorov, Z. L. Xiao, F. M. Peeters, and W. K. Kwok, Realization of Artificial Ice Systems for Magnetic
Vortices in a Superconducting MoGe Thin Film with Patterned Nanostructures, Phys. Rev. Lett. 111, 067001 (2013).

[24] C. Reichhardt and N. Grønbech-Jensen, Critical currents and vortex states at fractional matching fields in superconductors with periodic pinning, Phys. Rev. B 63, 054510 (2001).

[25] J. Trastoy, M. Malnou, C. Ulysse, R. Bernard, N. Bergeal, G. Faini, J. Lesueur, J. Briatico, and J. E. Villegas, Freezing and thawing of artificial ice by thermal switching of geometric frustration in magnetic flux lattices, Nat. Nanotechnol. 9, 710 (2014).

[26] I. A. Sadovskyy, Y. L. Wang, Z.-L. Xiao, W.-K. Kwok, and A. Glatz, Effect of hexagonal patterned arrays and defect geometry on the critical current of superconducting films, Phys. Rev. B 95, 075303 (2017).

[27] Y.-L. Wang, X. Ma, J. Xu, Z.-L. Xiao, A. Snezhko, R. Divan, L. E. Ocola, J. E. Pearson, B. Jánko, and W.-K. Kwok, Switchable geometric frustration in an artificial-spinice-superconductor heterosystem, Nat. Nanotechnol. 13, 560 (2018).

[28] C. Reichhardt and C. J. Olson, Novel Colloidal Crystalline States on Two-Dimensional Periodic Substrates, Phys. Rev. Lett. 88, 248301 (2002).

[29] T. Brazda, A. Silva, N. Manini, A. Vanossi, R. Guerra, E. Tosatti, and C. Bechinger, Experimental Observation of the Aubry Transition in Two-Dimensional Colloidal Monolayers, Phys. Rev. X 8, 011050 (2018).

[30] A. Ortiz-Ambriz and P. Tierno, Engineering of frustration in colloidal artificial ices realized on microfeatured grooved lattices, Nat. Commun. 7, 10575 (2016).

[31] R. L. Stoop, A. V. Straube, T. H. Johansen, and P. Tierno, Collective Directional Locking of Colloidal Monolayers on a Periodic Substrate, Phys. Rev. Lett. 124, 058002 (2020).

[32] C. Reichhardt and C. J. O. Reichhardt, Active matter commensuration and frustration effects on periodic substrates, Phys. Rev. E 103, 022602 (2021).

[33] M. Lewenstein, A. Sanpera, V. Ahufinger, B. Damski, A Sen(De), and U. Sen, Ultracold atomic gases in optical lattices: Mimicking condensed matter physics and beyond, Adv. Phys. 56, 243 (2007).

[34] Y. Huang, W. Li, C. Reichhardt, C. J. O. Reichhardt, and Y. Feng, Phonon spectra of a two-dimensional solid dusty plasma modified by two-dimensional periodic substrates, Phys. Rev. E 105, 015202 (2022).

[35] C. Reichhardt, D. Ray, and C. J. Olson Reichhardt, Quantized transport for a skyrmion moving on a two-dimensional periodic substrate, Phys. Rev. B 91, 104426 (2015).

[36] J. Feilhauer, S. Saha, J. Tobik, M. Zelent, L. J. Heyderman, and M. Mruczkiewicz, Controlled motion of skyrmions in a magnetic antidot lattice, Phys. Rev. B 102, 184425 (2020).

[37] C. Nisoli, R. Moessner, and P. Schiffer, Colloquium: Artificial spin ice: Designing and imaging magnetic frustration, Rev. Mod. Phys. 85, 1473 (2013).

[38] J. Hasnain, S. Jungblut, A. Tröster, and C. Dellago, Frictional dynamics of stiff monolayers: From nucleation dynamics to thermal sliding, Nanoscale 6, 10161 (2014).

[39] J. Koplik and G. Drazer, Nanoscale simulations of directional locking, Phys. Fluids 22, 052005 (2010).

[40] M. Çam, S. Lichter, and C. G. Goedde, Kink propagation and solute partitioning in an atomic monolayer on a substrate, Phys. Rev. E 104, L022801 (2021). 
[41] D. McDermott, J. Amelang, C. J. Olson Reichhardt, and C. Reichhardt, Dynamic regimes for driven colloidal particles on a periodic substrate at commensurate and incommensurate fillings, Phys. Rev. E 88, 062301 (2013).

[42] C. Reichhardt, C. J. Olson, and F. Nori, Nonequilibrium dynamic phases and plastic flow of driven vortex lattices in superconductors with periodic arrays of pinning sites, Phys. Rev. B 58, 6534 (1998).

[43] M. Durkin, I. Mondragon-Shem, S. Eley, T. L. Hughes, and N. Mason, History-dependent dissipative vortex dynamics in superconducting arrays, Phys. Rev. B 94, 024510 (2016).

[44] M. B. Hastings, C. J. Olson Reichhardt, and C. Reichhardt, Depinning by Fracture in a Glassy Background, Phys. Rev. Lett. 90, 098302 (2003).

[45] P. Habdas, D. Schaar, A. C. Levitt, and E. R. Weeks, Forced motion of a probe particle near the colloidal glass transition, Europhys. Lett. 67, 477 (2004).

[46] T. M. Squires and J. F. Brady, A simple paradigm for active and nonlinear microrheology, Phys. Fluids 17, 073101 (2005).

[47] Th. Voigtmann and M. Fuchs, Force-driven micro-rheology, Eur. Phys. J. Spec. Top. 222, 2819 (2013).

[48] R. N. Zia, Active and passive microrheology: Theory and simulation, Ann. Rev. Fluid Mech. 50, 371 (2018).

[49] I. Gazuz, A. M. Puertas, Th. Voigtmann, and M. Fuchs, Active and Nonlinear Microrheology in Dense Colloidal Suspensions, Phys. Rev. Lett. 102, 248302 (2009).

[50] C. Reichhardt and C. J. O. Reichhardt, Dynamics and nonmonotonic drag for individually driven skyrmions, Phys. Rev. B 104, 064441 (2021).

[51] D. Winter, J. Horbach, P. Virnau, and K. Binder, Active Nonlinear Microrheology in a Glass-Forming Yukawa Fluid, Phys. Rev. Lett. 108, 028303 (2012).

[52] N. Şenbil, M. Gruber, C. Zhang, M. Fuchs, and F. Scheffold, Observation of Strongly Heterogeneous Dynamics at the Depinning Transition in a Colloidal Glass, Phys. Rev. Lett. 122, 108002 (2019).

[53] J. W. Yu, S. H. E. Rahbari, T. Kawasaki, H. Park, and W. B. Lee, Active microrheology of a bulk metallic glass, Sci. Adv. 6, 29 (2020).

[54] J. A. Drocco, M. B. Hastings, C. J. Olson Reichhardt, and C. Reichhardt, Multiscaling at Point $J$ : Jamming is a Critical Phenomenon, Phys. Rev. Lett. 95, 088001 (2005).

[55] R. Candelier and O. Dauchot, Journey of an intruder through the fluidization and jamming transitions of a dense granular media, Phys. Rev. E 81, 011304 (2010).

[56] E. Kolb, P. Cixous, N. Gaudouen, and T. Darnige, Rigid intruder inside a two-dimensional dense granular flow: Drag force and cavity formation, Phys. Rev. E 87, 032207 (2013).

[57] R. P. A. Dullens and C. Bechinger, Shear Thinning and Local Melting of Colloidal Crystals, Phys. Rev. Lett. 107, 138301 (2011).

[58] C. Abaurrea-Velasco, C. Lozano, C. Bechinger, and J. de Graaf, Autonomously Probing Viscoelasticity in Disordered Suspensions, Phys. Rev. Lett. 125, 258002 (2020).

[59] E. W. J. Straver, J. E. Hoffman, O. M. Auslaender, D. Rugar, and K. A. Moler, Controlled manipulation of individual vortices in a superconductor, Appl. Phys. Lett. 93, 172514 (2008).
[60] C. Reichhardt, Vortices wiggled and dragged, Nat. Phys. 5, 15 (2009).

[61] O. M. Auslaender, L. Luan, E. W. J. Straver, J. E. Hoffman, N. C. Koshnick, E. Zeldov, D. A. Bonn, R. Liang, W. N. Hardy, and K. A. Moler, Mechanics of individual isolated vortices in a cuprate superconductor, Nat. Phys. 5, 35 (2009).

[62] C. Hanneken, A. Kubetzka, K. von Bergmann, and R. Wiesendanger, Pinning and movement of individual nanoscale magnetic skyrmions via defects, New J. Phys. 18, 055009 (2016).

[63] A. Casiraghi, H. Corte-León, M. Vafaee, F. Garcia-Sanchez, G. Durin, M. Pasquale, G. Jakob, M. Kläui, and O. Kazakova, Individual skyrmion manipulation by local magnetic field gradients, Commun. Phys. 2, 145 (2019).

[64] N. Shapira, Y. Lamhot, O. Shpielberg, Y. Kafri, B. J. Ramshaw, D. A. Bonn, R. Liang, W. N. Hardy, and O. M. Auslaender, Disorder-induced power-law response of a superconducting vortex on a plane, Phys. Rev. B 92, 100501(R) (2015).

[65] I. S. Veshchunov, W. Magrini, S. V. Mironov, A. G. Godin, J. B. Trebbia, A. I. Buzdin, Ph. Tamarat, and B. Lounis, Optical manipulation of single flux quanta, Nat. Commun. 7, 12801 (2016).

[66] A. Kremen, S. Wissberg, N. Haham, E. Persky, Y. Frenkel, and B. Kalisky, Mechanical control of individual superconducting vortices, Nano Lett. 16, 1626 (2016).

[67] H. Polshyn, T. Naibert, and R. Budakian, Manipulating multivortex states in superconducting structures, Nano Lett. 19, 5476 (2019).

[68] C. J. Olson Reichhardt and C. Reichhardt, Viscous decoupling transitions for individually dragged particles in systems with quenched disorder, Phys. Rev. E 78, 011402 (2008).

[69] X. Ma, C. J. O. Reichhardt, and C. Reichhardt, Manipulation of individual superconducting vortices and stick-slip motion in periodic pinning arrays, Phys. Rev. B 97, 214521 (2018).

[70] X. Ma, C. J. O. Reichhardt, and C. Reichhardt, Braiding Majorana fermions and creating quantum logic gates with vortices on a periodic pinning structure, Phys. Rev. B 101, 024514 (2020).

[71] C. Reichhardt and C. J. Olson Reichhardt, Commensurability effects at nonmatching fields for vortices in diluted periodic pinning arrays, Phys. Rev. B 76, 094512 (2007).

[72] C. Reichhardt and C. J. Olson Reichhardt, Transport anisotropy as a probe of the interstitial vortex state in superconductors with artificial pinning arrays, Phys. Rev. B 79, 134501 (2009).

[73] N. Poccia, T. I. Baturina, F. Coneri, C. G. Molenaar, X. R. Wang, G. Bianconi, A. Brinkman, H. Hilgenkamp, A. A. Golubov, and V. M. Vinokur, Critical behavior at a dynamic vortex insulator-to-metal transition, Science 349, 1202 (2015).

[74] Z. Jiang, D. A. Dikin, V. Chandrasekhar, V. V. Metlushko, and V. V. Moshchalkov, Pinning phenomena in a superconducting film with a square lattice of artificial pinning centers, Appl. Phys. Lett. 84, 5371 (2004).

[75] C. Reichhardt and F. Nori, Phase Locking, Devil's Staircases, Farey Trees, and Arnold Tongues in Driven Vortex Lattices with Periodic Pinning, Phys. Rev. Lett. 82, 414 (1999).

[76] P. T. Korda, M. B. Taylor, and D. G. Grier, Kinetically Lockedin Colloidal Transport in an Array of Optical Tweezers, Phys. Rev. Lett. 89, 128301 (2002). 